Annales Geophysicae (2002) 20: 1577-1601 (c) European Geosciences Union 2002

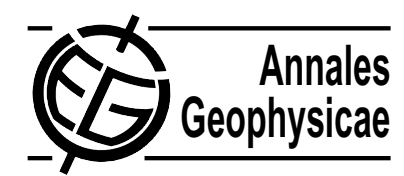

\title{
Excitation of twin-vortex flow in the nightside high-latitude ionosphere during an isolated substorm
}

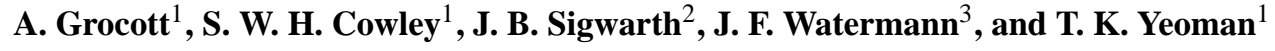 \\ ${ }^{1}$ Department of Physics and Astronomy, University of Leicester, Leicester LE1 7RH, UK \\ ${ }^{2}$ Department of Physics and Astronomy, The University of Iowa, Iowa City, Iowa 52242-1479, USA \\ ${ }^{3}$ Danish Meteorological Institute, Lyngbyvej 100, 2100 Copenhagen, Denmark
}

Received: 25 June 2001 - Revised: 26 March 2002 - Accepted: 29 March 2002

\begin{abstract}
We present SuperDARN radar observations of the ionospheric flow during a well-observed high-latitude substorm which occurred during steady northward IMF conditions on 2 December 1999. These data clearly demonstrate the excitation of large-scale flow associated with the substorm expansion phase, with enhanced equatorward flows being observed in the pre-midnight local time sector of the expansion phase auroral bulge and westward electrojet, and enhanced return sunward flows being present at local times on either side, extending into the dayside sector. The flow pattern excited was thus of twin-vortex form, with foci located at either end of the substorm auroral bulge, as imaged by the Polar VIS UV imager. Estimated total transpolar voltages were $\sim 40 \mathrm{kV}$ prior to expansion phase onset, grew to $\sim 80 \mathrm{kV}$ over a $\sim 15$ min interval during the expansion phase, and then decayed to $\sim 35 \mathrm{kV}$ over $\sim 10 \mathrm{~min}$ during recovery. The excitation of the large-scale flow pattern resulted in the development of magnetic disturbances which extended well outside of the region directly disturbed by the substorm, depending upon the change in the flow and the local ionospheric conductivity. It is estimated that the nightside reconnection rate averaged over the 24-min interval of the substorm was $\sim 65$ $75 \mathrm{kV}$, compared with continuing dayside reconnection rates of $\sim 30-45 \mathrm{kV}$. The net closure of open flux during the substorm was thus $\sim 0.4-0.6 \times 10^{8} \mathrm{~Wb}$, representing $\sim 15-20 \%$ of the open flux present at onset, and corresponding to an overall contraction of the open-closed field line boundary by $\sim 1^{\circ}$ latitude.
\end{abstract}

Key words. Ionosphere (auroral ionosphere; ionospheremagnetosphere interactions; plasma convection)

\section{Introduction}

According to the theoretical picture proposed by Cowley and Lockwood (1992), Dungey-cycle flow in the Earth's coupled solar wind-magnetosphere-ionosphere system may be con-

Correspondence to: A. Grocott (ag27@ion.le.ac.uk) sidered to be excited principally by two essentially independent processes. The first is reconnection at the magnetopause between the Earth's field and the interplanetary field in the magnetosheath, which results in the transfer of newly-formed open flux tubes from the dayside into the tail lobes. This process causes a departure of the system from equilibrium, and excites flow which carries the system towards a new equilibrium configuration with the changed (increased) amount of open flux. In the ionosphere, the flow is of twin-vortex form, with antisunward flow over the polar cap and return sunward flow at lower latitudes. The flow is expected to be strongest on the dayside, with the foci of the twin-vortex flow located at either end of the dayside merging gap, and is associated with an expansion in size of the open polar cap and surrounding auroral zone.

The second process is the reconnection of open tail lobe flux across the tail current sheet, which results in the destruction of open lobe flux, and the contraction of newly-formed closed flux tubes towards the Earth. This process similarly perturbs the system from equilibrium, and also excites flow which is of twin-vortex form in the ionosphere. Now, however, the flow will be strongest on the nightside, with the foci of the twin-vortex flow located at either end of the nightside merging gap, and is associated with a contraction of the open polar cap and surrounding auroral zone. This two-component flow picture, while first being presented in the above form by Cowley and Lockwood (1992), is related to earlier ideas on boundary motions and flows presented by Siscoe and Huang (1985) and Freeman and Southwood (1988). Further discussion may be found in the more recent papers by Cowley and Lockwood (1996) and Cowley (1998).

The excitation of flow in the dayside ionosphere during intervals of southward interplanetary magnetic field (IMF) and consequent magnetopause reconnection is now a wellestablished phenomenon (e.g. Etemadi et al., 1988; Todd et al., 1988; Ruohoniemi et al., 1993; Ridley et al., 1998; Khan and Cowley, 1999). Even the substructure of characteristically pulsed dayside reconnection on 5-10 min time scales (i.e. flux transfer events) are well-resolved in dayside flow 
data as pulsed ionospheric flows (Lockwood et al., 1989, 1993; Elphic et al., 1990; Pinnock et al., 1993, 1995; Moen et al., 1995; Provan et al., 1998; Neudegg et al., 1999, 2000; Milan et al., 2000; Wild et al., 2001). As a consequence, there is now little uncertainty about the central role of magnetopause reconnection in magnetospheric dynamics and flow excitation.

The same cannot be said, however, of the corresponding relationship between tail reconnection and flow, which has recently been discussed in more detail by Cowley et al. (1998) and Lu et al. (2001). Two distinct phenomena have been linked to tail reconnection. The first of these relates to azimuthally-localized impulsive events in which auroras are first intensified at the poleward boundary of the nightside auroral zone, and then expand equatorward, reaching to near the equatorward boundary of the oval emissions after $\sim 5$ min (Henderson et al., 1998). These events, termed poleward boundary intensifications (PBIs) by Lyons et al. (1999), have been found to occur in all phases of the substorm cycle, including during long intervals of magnetic quiet, though they appear to be more frequent during substorm expansion phases. They are associated with azimuthally-localized bursty bulk flows (BBFs) in the near-Earth plasma sheet (Baumjohann et al., 1990; Angelopoulos et al., 1992; Yeoman et al., 1998; Lyons et al., 1999) and the excitation of equatorward flow in the ionosphere (Sergeev et al., 1990; de la Beaujardire et al., 1994; Yeoman and Lühr, 1997; Watanabe et al., 1998), together with relatively weak structured magnetic disturbances in the high-latitude ionosphere, Pi2 signals, and particle injections at geosynchronous orbit (Hsu and McPherron, 1996; Henderson et al., 1998; Lyons et al., 1999; Sergeev et al., 1999, 2000). As discussed, for example, by Cowley (1998), and Cowley et al. (1998), these features are strongly suggestive of the occurrence of localized impulsive reconnection in the tail leading to plasma injection and flow excitation. During quiet times, at least, the reconnection site is presumed to be that lying in the more distant magnetotail beyond $\sim 100 \mathrm{R}_{\mathrm{E}}$.

While PBI/BBF events thus probably represent the consequences of localized impulsive reconnection in the tail, which can occur under a wide variety of conditions, it has long been supposed that major episodes of tail reconnection and open flux destruction take place during magnetospheric substorms (Hones, 1979; Baker et al., 1996). Controversy still surrounds the sequence of events which occur during the few-minute phase which initiates substorm expansion, with some observations suggesting that tail reconnection is initiated by near-Earth current sheet disruption followed by tailward-propagating inward collapse of the field lines (e.g. Lui, 1991, 1996; Frank and Sigwarth, 2000; Friedrich et al., 2001). Nevertheless, recent results from the Geotail spacecraft do show that reconnection typically begins in the dusk sector plasma sheet at down-tail distances of $\sim 20-30 R_{E}$, a few minutes before the onset of expansion signatures on the ground, and then expands to encompass a significant fraction of the dusk and midnight sector tail (Nagai et al., 1998; Nagai and Machida, 1998; Petrukovich et al., 1998; Machida et al., 1999). Both initial dipolarization and the convection that results from tail reconnection contribute to substorm-associated Dungey-cycle flux transport in the tail. This being so, we would expect that significant largescale twin-vortex flows should be excited during substorms, with the reconnection-associated transport presumably dominating on longer expansion phase time scales (few tens of minutes), corresponding in essence to the reconnectionassociated flows observed on the dayside cited above.

Observational evidence for such flow excitation, however, has at best been ambiguous. In a recent study of Sondrestrom radar data, Blanchard et al. (1997) (following earlier work by de la Beaujardière et al., 1991 and Blanchard et al., 1996) measured the local reconnection rate by examining the ionospheric flow across the open-closed field line boundary, the position of the latter being estimated from ionospheric features observed by the radar and $630 \mathrm{~nm}$ auroral emission. They found that an increase in the reconnection rate occurs near midnight, shortly after expansion phase onset, expanding to encompass a wider local time sector after $\sim 20 \mathrm{~min}$. However, the initial reconnection rate increase was manifest mainly in a poleward motion of the inferred open-closed field line boundary. Flows were found to become elevated only after an interval of $\sim 30 \mathrm{~min}$. Somewhat correspondingly, Weimer (1999) has presented a statistical survey of low-altitude electric field data from the DE-2 spacecraft, separated according to the IMF direction and into substorm and non-substorm intervals. The flow patterns for a given IMF orientation show a more pronounced Harang asymmetry on the nightside during substorm intervals than during non-substorm intervals, but the total transpolar voltage values showed little overall difference. On the other hand, Opgenoorth and Pellinen (1998), following earlier work by Grafe et al. (1987), have presented evidence for flow enhancements in the dusk auroral zone immediately following expansion phase onset in the midnight sector, leading to an immediate increase in the global directly driven current system. They suggest, however, that diversion of the twin-vortex flow around the low-flow high-conductivity auroral bulge formed during the expansion phase (e.g. Kirkwood et al., 1988; Morelli et al., 1995; Yeoman et al., 2000; Khan et al., 2001) may play a role. Fox et al. (1999) have also reported observations of a surge of transpolar flow into the midnight sector associated with a substorm intensification, which they suggest was due to a burst of reconnection in the tail. Most recently, Grocott et al. (2000) have presented an analysis of SuperDARN radar flow data obtained during an isolated substorm, and have found evidence for the excitation of twin-vortex flow cells centered in the nightside ionosphere, which enhance the transpolar voltage by $\sim 20 \mathrm{kV}$ compared with pre-onset values.

Given these somewhat varied conclusions, taken together with rather clear theoretical expectations, further study of the important issue of large-scale ionospheric flow excitation during substorms is clearly warranted. The international SuperDARN HF radar network employed by Grocott et al. (2000), which is now able to image the ionospheric flow over 
a large fraction of the polar regions with good time resolution (Greenwald et al., 1995; Shepherd and Ruohoniemi, 2000) is clearly the most suitable tool to undertake this task. Nevertheless, the intervention of several factors introduces significant difficulties into this undertaking. The first is that substorm intervals are usually associated with periods of large and variable negative IMF $B_{z}$, which will result in large variations of the ionospheric flow through modulated magnetopause reconnection. These variations may be expected to be at least as large as those associated with nightside reconnection and substorms, such that the flow variations associated with the substorms may be obscured. Second, substorms are generally initiated in the ionosphere on field lines mapping to $\sim 65^{\circ}$. This latitude is close to the equatorward edge of the SuperDARN field of view (in normal scan mode), so that the substorm-effected region may only be partially imaged. Third, it is very often the case that SuperDARN radar backscatter drops out during intervals of magnetic disturbance due to precipitation-induced D-region radio absorption (Milan et al., 1996; Yeoman and Lühr, 1997). In this case the evolution of the flow during the substorm cannot be properly followed after the onset of the expansion phase.

In this paper we study the flows which occurred during an isolated substorm on 2 December 1999 under somewhat fortuitous circumstances, such that the above difficulties were largely circumvented. First, the substorm occurred during an interval of very steady northward IMF (and large negative $B_{y}$ ), such that the flows driven from the dayside may be expected to have been steady in character and modest in magnitude. Second, due to these overall relatively quiet conditions, the substorm occurred at higher latitudes than usual, with the electrojet centered near $\sim 70^{\circ}$, and was thus located well within the region of usual SuperDARN coverage. Furthermore, the substorm occurred in the pre-midnight sector centrally within the region viewed by the six Northern Hemisphere radars operating at that time, so that the flows both within the substorm-disturbed region, and in the regions both to its east and west, were well monitored during the disturbance. Third, backscatter was observed throughout the interval essentially across the full range of local times covered by the radars (though still dropping out within part of the substorm bulge region itself), so that good estimates could be made of the overall voltage associated with the flow. This was probably due to the high-latitude nature of the substorm, such that the enhanced D-region lay poleward of where the HF radar beams traversed that layer of the ionosphere. These observations demonstrate unequivocally an excitation of twin-vortex flow associated with the substorm expansion phase, with enhanced equatorward flows occurring in the local time sector of the bulge, and enhanced return sunward flows being present at earlier and later local times. We find that the transpolar voltage increased in the expansion phase from $\sim 40 \mathrm{kV}$, during the pre-onset interval to peak at $\sim 80 \mathrm{kV}$ after $\sim 15 \mathrm{~min}$, and then died away over $\sim 10$ min during the recovery phase to $\sim 35 \mathrm{kV}$.

\section{Instrumentation}

In this section we provide brief details of the instrumentation employed in this study, which principally concerns the interval 00:20-01:40 UT on 2 December 1999.

\subsection{SuperDARN radar data}

The central focus of our study is the measurement of ionospheric convection velocities provided by the Super Dual Auroral Radar Network (SuperDARN). SuperDARN is an international array of HF coherent radars spanning the auroral regions of both the Northern and Southern Hemispheres (Greenwald et al., 1995). At the time of the interval described here, the Northern Hemisphere network consisted of six radars, whose fields of view covered approximately $12 \mathrm{~h}$ of magnetic local time (MLT). This is illustrated in Fig. 1a, where we show a view looking onto the northern magnetic pole. With increasing MLT we show the fields of view of the Saskatoon (T), Kapuskasing (K), Goose Bay (G), Iceland West (W), Iceland East (E), and Finland (F) radars. These are shown on a geomagnetic grid at 01:00 UT (a central time during the study, corresponding to maximum magnetic/flow disturbance during the substorm), extending from the pole to $60^{\circ}$ latitude, with noon at the top and dusk to the left. The coordinates are Altitude Adjusted Corrected Geomagnetic (AACGM) coordinates, a development of the PACE system discussed by Baker and Wing (1989). The fieldsof-view of the three radars whose data will be displayed in detail are shaded blue, with data being displayed from the beam directions marked by the red lines.

All six radars were operating in a standard mode during the study interval, in which each radar scans through 16 beams of azimuthal separation $3.24^{\circ}$, with a total scan time of $2 \mathrm{~min}$. Each beam is divided into 75 range gates of length $45 \mathrm{~km}$, and so in each full scan the radar covers $52^{\circ}$ in azimuth and over $3000 \mathrm{~km}$ in range. Large-scale maps of the high-latitude convection have been derived from this data using the MapPotential model developed by Ruohoniemi and Baker (1998). In this model, the line-of-sight velocities are mapped onto a polar grid, and used to determine a solution for the electrostatic potential, which is expressed in spherical harmonics. The equipotentials of the solution then represent the plasma streamlines of the modelled convection pattern. Information from the statistical model of Ruohoniemi and Greenwald (1996), parameterized by concurrent IMF conditions, is used to stabilize the solution where no measurements are available. In this paper, spherical harmonic fits using terms up to sixth order are employed to fit the radar data, as deemed appropriate from the good overall coverage of the data.

\subsection{Ground magnetometer data}

In Fig. 1b we show the locations of the ground magnetic stations employed, on the same magnetic grid at 01:00 UT as in Fig. 1a. Measurements are provided by magnetometers from six Northern Hemisphere arrays which span the same 


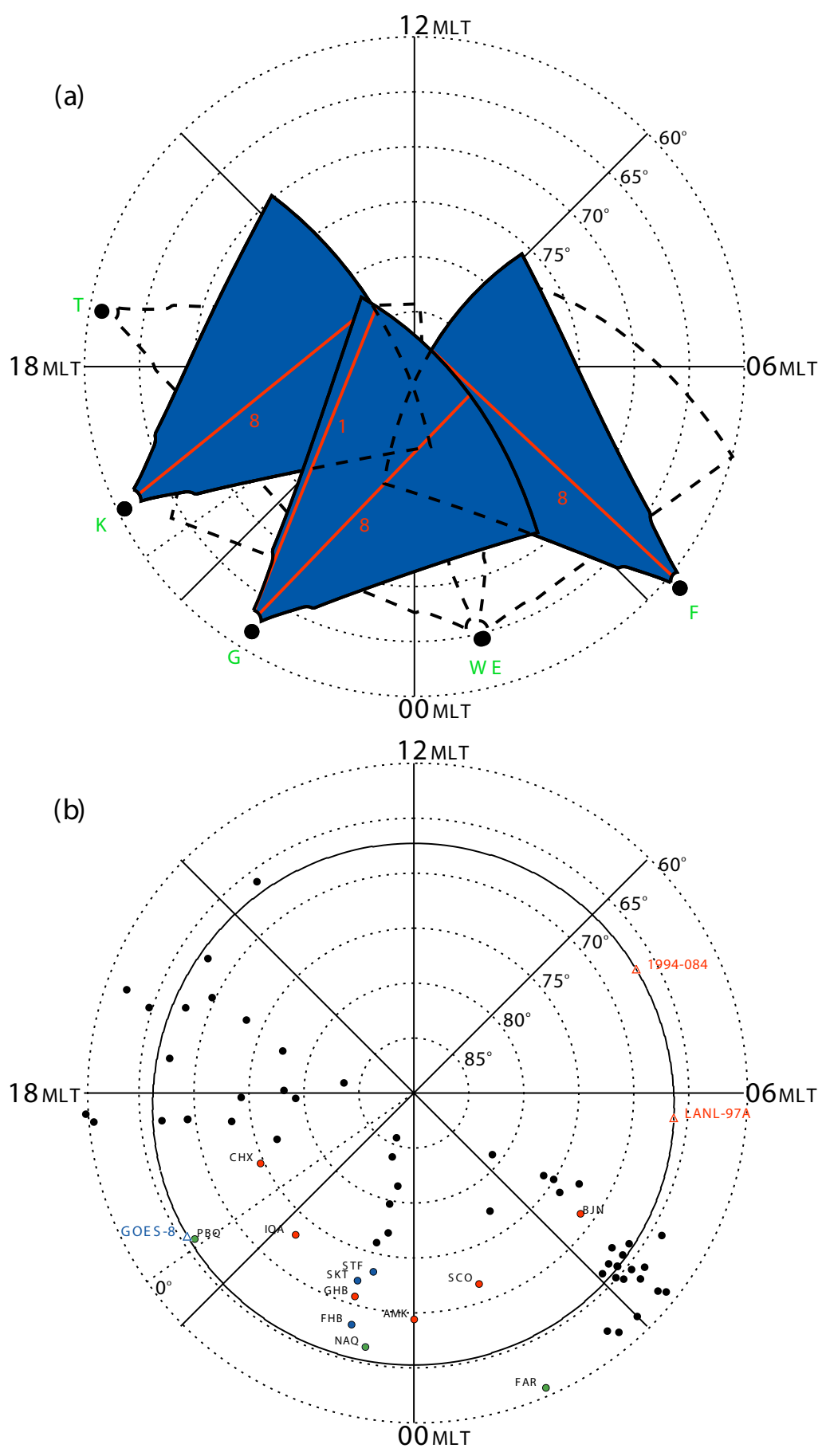

Fig. 1. (a) Fields-of-view of the six Northern Hemisphere SuperDARN radars at 01:00 UT, plotted on a geomagnetic grid from the pole to $60^{\circ}$, with 12 MLT at the top and dusk to the left. Fields-of-view coloured blue are those of the three radars for which detailed line-of-sight measurements will be discussed, using the beams indicated in red. (b) Shows ground-based magnetometer locations and geostationary spacecraft footprints at 01:00 UT on the same grid. Coloured magnetometer locations are those for which timeseries plots will be shown. Green dots indicate stations whose data has been band-pass filtered to provide information on Pi2 signals. Red dots indicate stations which form a longitude chain at $\sim 70^{\circ}-75^{\circ}$, which define the local time extent of the principal magnetic disturbance. Blue dots indicate stations of the Greenland west coast latitude chain (additional to GHB and NAQ which are coloured red and green as just indicated), which define the latitudinal extent of the main magnetic disturbance. The black oval represents the ionospheric footprint of geostationary orbit (specifically that of the GOES-8 spacecraft). On it are plotted the locations of the GOES-8, LANL-97A, and 1994-084 spacecraft.

$12 \mathrm{~h}$ of MLT as the SuperDARN radars. These are the Canadian CANOPUS array (Grant et al., 1992), the Geological Survey of Canada array, the MACCS array (Hughes and Engebretson, 1997), the Greenland Magnetometer Chain (FriisChristensen et al., 1985), the Scandinavian IMAGE array (Lühr et al., 1998), and the SAMNET array (Yeoman et al., 1990). Time-series data from all six chains will be presented in $H, D, Z$ coordinates, where $H$ is local magnetic north, $D$ is local magnetic east, and $Z$ is vertically down.
The identifier codes of specific magnetometer stations which are of particular interest are also indicated in the figure. Data from those at lower latitudes, colour-coded green (i.e. PBQ, NAQ, and FAR), have been band-pass filtered (20$200 \mathrm{~s}$ ) to provide information on $\mathrm{Pi} 2$ signals. Those at latitudes $\sim 70^{\circ}-75^{\circ}$, colour-coded red (i.e. CHX, IQA, GHB, AMK, SCO, and BJN), define the local time extent of the principal magnetic disturbances during the substorm, spanning $\sim 8 \mathrm{~h}$ of MLT from post-dusk, through midnight, to pre- 
dawn. In addition, we will show data from the Greenland west coast latitudinal chain STF, SKT, GHB, FHB, and NAQ, of which GHB and NAQ are colour-coded red and green as just indicated, while the remainder are colour-coded blue. This chain spans the substorm electrojet between $\sim 66^{\circ}$ and $\sim 74^{\circ}$ magnetic latitude.

\subsection{Geostationary spacecraft data}

The coloured triangles in Fig. 1b show the ionospheric footprints at 01:00 UT of three geostationary spacecraft, whose data are employed in this study to verify the occurrence of normal substorm phenomena. These have been mapped from geostationary orbit using the T-89 model for $K_{p}=2$ (Tsyganenko, 1989). This is the $K_{p}$ value prevailing during the interval 00:00-03:00 UT. The blue triangle shows the footprint of GOES-8, located (at 01:00 UT) in the pre-midnight sector just to the west (and equatorward of) the main substorm disturbed region. The black solid line shows the mapped orbit of this spacecraft. Magnetometer data from GOES-8 will be used to characterize the nightside geostationary magnetic field during the interval (Singer et al., 1996). The red triangles show the positions of Los Alamos National Laboratory spacecraft LANL-97A and 1994-084. The SOPA instruments carried by each spacecraft provide high-resolution energetic particle measurements (Belian et al., 1992). Here we will use these data to examine the injection of energetic ions and electrons into geostationary orbit during the substorm.

\subsection{Polar auroral images}

Images of the aurora were provided during the interval by the VIS imager on the Polar spacecraft (Frank et al., 1995). This instrument viewed the whole of the northern polar ionosphere during the course of the substorm, and provided images approximately every $5 \mathrm{~min}$. These consist of $32.5 \mathrm{~s}$ integrations of UV photons at a wavelength of $130.4 \mathrm{~nm}$.

\subsection{Upstream interplanetary parameters}

Interplanetary conditions were monitored by the ACE spacecraft (Stone et al., 1998), located upstream of the Earth at GSM coordinates $(X, Y, Z)=(+221,+21,-25) R_{E}$. The variation of the spacecraft position over the interval was not significant. Solar wind and IMF data were obtained by the SWEPAM and MAG instruments, respectively (McComas et al., 1998; Smith et al., 1999). The propagation delay of field changes from ACE to the dayside ionosphere has been estimated using the method of Khan and Cowley (1999). The estimate includes the propagation time in the solar wind upstream of the bow shock, the frozen-in transit time across the subsolar magnetosheath, and the Alfvénic propagation time along open field lines from the subsolar magnetopause to the ionosphere. With solar wind densities $\sim 12 \mathrm{~cm}^{-3}$ and speeds $\sim 350 \mathrm{~km} / \mathrm{s}$, we find a propagation delay of $74 \pm 5 \mathrm{~min}$, and this time has been used to lag the ACE interplanetary data displayed here.

\section{Observations}

In this section we discuss the ground and space observations made during the substorm. We begin with an overview of the interplanetary and geomagnetic conditions during an expanded interval surrounding the substorm, and subsequently look in more detail at the magnetic, flow, and auroral data during the substorm itself.

\subsection{Upstream interplanetary conditions}

Figure 2a presents data from the ACE spacecraft, lagged by 74 min as described, during the 10-h interval from 19:00 UT on 1 December to 05:00 UT on 2 December 1999. The vertical dashed lines on this and subsequent figures indicate the onset time of the substorm expansion at 00:44 UT, and the beginning of the recovery phase at 01:06 UT. Details of the determination of these times will be discussed in Sect. 3.3. The top two panels show the solar wind proton number density $\left(N_{p}\right)$ and speed $\left(V_{p}\right)$ from ACE/SWEPAM (for which data were available only up to 03:30 UT lagged time). During this interval the density varied between 5 and $20 \mathrm{~cm}^{-3}$, and the velocity between 330 and $380 \mathrm{~km} / \mathrm{s}$. However, it is apparent that during the substorm interval both the density and velocity were relatively steady at $\sim 12 \mathrm{~cm}^{-3}$ and $\sim 350 \mathrm{~km} / \mathrm{s}$, respectively. The bottom three panels illustrate the three components of the IMF in GSM coordinates from ACE/MAG. Throughout most of the interval, $B_{x}$ remained small and negative, typically 0 to $-5 \mathrm{nT}$, while $B_{y}$ was large and negative, around $-10 \mathrm{nT}$. $B_{z}$ was also negative until $\sim 20: 50$ UT (lagged time), thereafter remaining close to zero until $\sim 00: 10$ UT. Between $\sim 00: 10$ UT and $\sim 02: 15$ UT, $B_{z}$ was consistently northward, and remained at a constant $+5 \mathrm{nT}$ during the substorm expansion phase. At $\sim 02: 15 \mathrm{UT}$ it underwent a brief southward excursion, before returning northward for the remainder of the interval.

\subsection{Ground magnetic data overview}

Figure $2 \mathrm{~b}$ presents $H$ component measurements from the six ground magnetometers which comprise the $\sim 120^{\circ}$ longitudinal array coloured red in Fig. 1b. The vertical arrows indicate magnetic local midnight for each of the stations (midnight for CHX occurs at 05:26 UT, just beyond the interval displayed). The horizontal dotted lines are baselines for the magnetic disturbance observed at each station, the choice of which will be discussed in Sect. 3.6. An initial magnetic disturbance starts just after 19:00 UT, coinciding with the interval of negative IMF $B_{z}$ mentioned above. However, these perturbations start to decay simultaneously with the northward turning of the IMF at $\sim 20: 50 \mathrm{UT}$, with all traces returning to near baseline by $\sim 00: 00 \mathrm{UT}$. Then, at $\sim 00: 44 \mathrm{UT}$ (first dashed line) a sharp negative bay onset begins in the midnight sector, with small negative precursory activity starting at $\sim 00: 35$ UT. A peak disturbance of $-275 \mathrm{nT}$ was observed at AMK at 00:55 UT. UV imagery to be shown below demonstrate that the main auroral disturbance spanned 
(a)

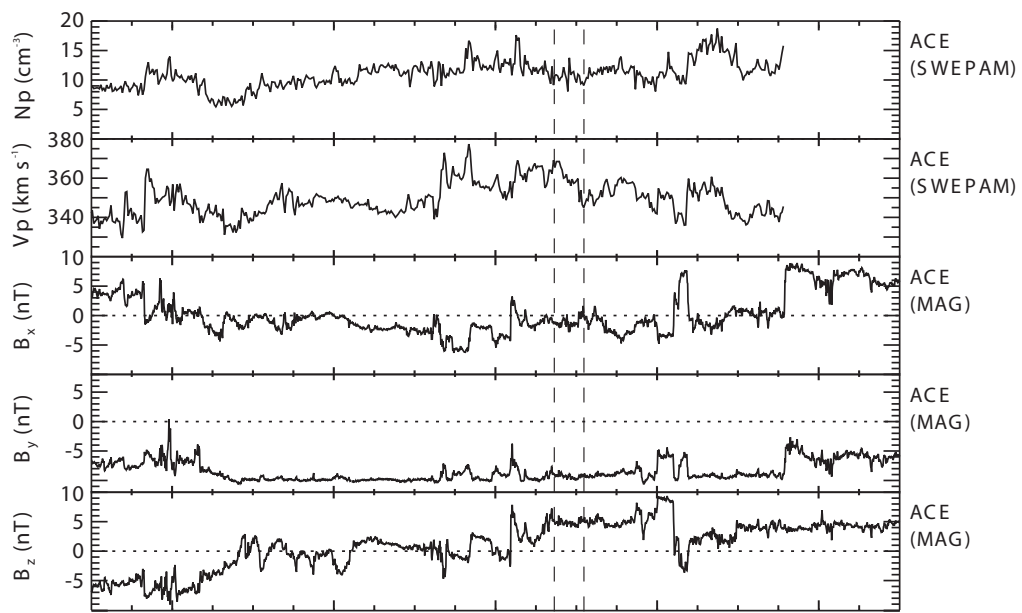

(b)
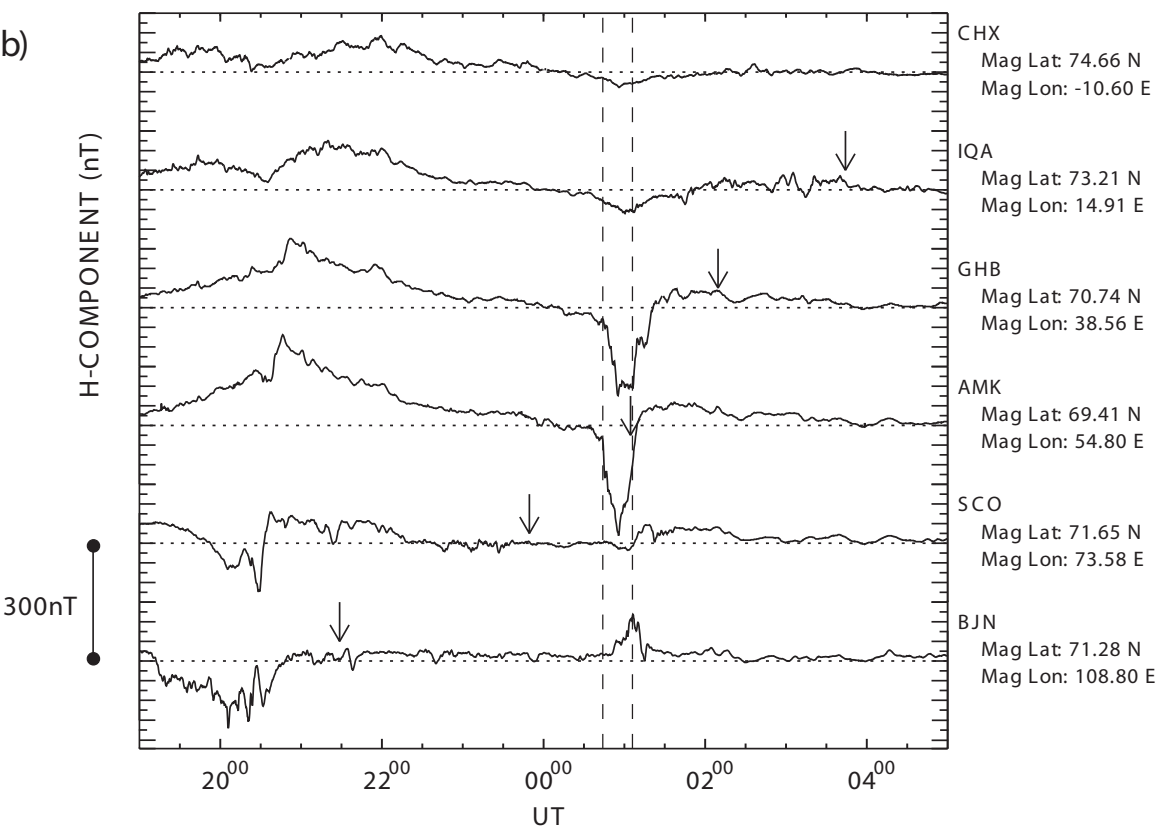

Fig. 2. (a) Upstream interplanetary observations from the ACE spacecraft, lagged by $74 \mathrm{~min}$ to account for the propagation delay to the ionosphere. The top two panels show solar wind density and velocity data, while the bottom three panels show IMF data in GSM coordinates, for 19:00 UT on 1 December to 05:00 UT on 2 December 1999. The times of substorm expansion phase onset and recovery onset are marked with vertical dashed lines. (b) Shows $H$-component magnetic field measurements from the azimuthal magnetometer array shown by the red dots in Fig. 1b. The separation between magnetometer baselines (horizontal dotted lines) is $300 \mathrm{nT}$. These baselines have been taken as the field measured at each station at 04:00 UT, as discussed in the text. Vertical arrows indicate the time of magnetic local midnight. Magnetic midnight for station CHX occurs at $\sim$ 05:26 UT, just beyond the interval displayed. the interval from $\sim 20: 00$ to $\sim 01: 00$ MLT, thus encompassing stations IQA, GHB, and AMK (located at 21:16, 22:50, and 23:55 MLT at 01:00 UT). Figure $2 \mathrm{~b}$ demonstrates that magnetic perturbations were also observed outside this region, with weak negative disturbances peaking at $-50 \mathrm{nT}$ being observed at CHX at $\sim 19: 30$ MLT, variable fields of $\sim 75 \mathrm{nT}$ amplitude at SCO at $\sim 01: 10 \mathrm{MLT}$, and positive perturbations peaking at $125 \mathrm{nT}$ at BJN at $\sim 03: 30$ MLT. Thus, as previously emphasized by Opgenoorth and Pellinen (1998), magnetic disturbances occur during the expansion phase over a broad range of MLT (greater than $8 \mathrm{~h}$ in this case), and are not confined to the range of local times occupied by the auroral bulge. These disturbances will be related below to changes in the flow pattern that occur during the substorm. An equally rapid bay recovery began at $\sim 01: 06 \mathrm{UT}$ (second vertical dashed line). The main interval of disturbance was concluded by $\sim 01: 20 \mathrm{UT}$, with more gradual variations towards baseline values occurring after that.

\subsection{Greenland west magnetic data}

We now examine data from the substorm-disturbed region itself. In Fig. 3 we present magnetic measurements from the meridional array of west coast Greenland magnetometers (the blue array in Fig. 1b). These were located near 23:00 MLT, and thus centrally within the substorm-disturbed region at the time of substorm onset. We show data for an 80-min interval spanning the substorm, from 00:20 to 01:40 UT on 2 December. The vertical dashed lines show the times of onset of the expansion and recovery phases as in Fig. 2. Panel (i) shows the $H$ component at stations NAQ to STF, spanning magnetic latitudes from $\sim 66^{\circ}$ to $\sim 73^{\circ}$. The largest negative disturbance occurred at GHB at $\sim 71^{\circ}$, reaching $\sim-225 \mathrm{nT}$ at 00:55 UT. Consistently smaller values were observed both poleward and equatorward, while only positive perturbations were observed at NAQ at $\sim 66^{\circ}$. These data thus suggest that the centre of the westward elec- 
(i)

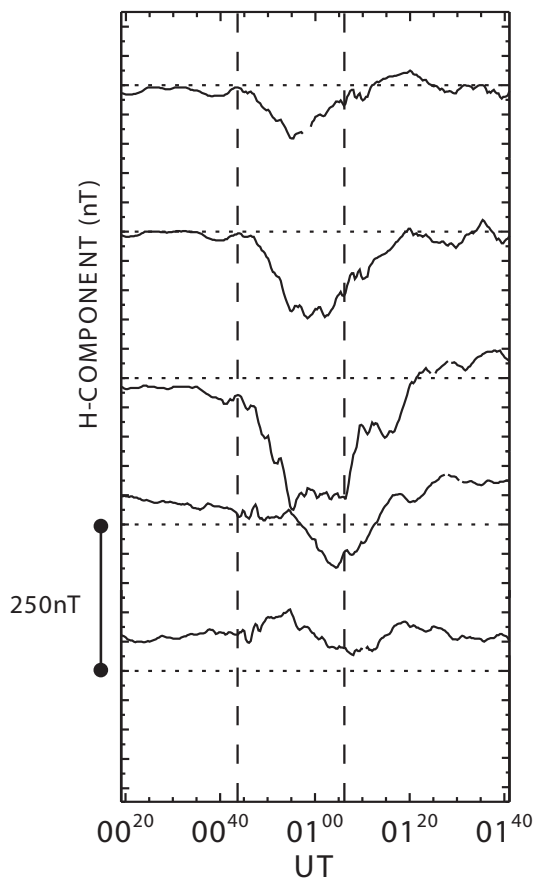

(ii)

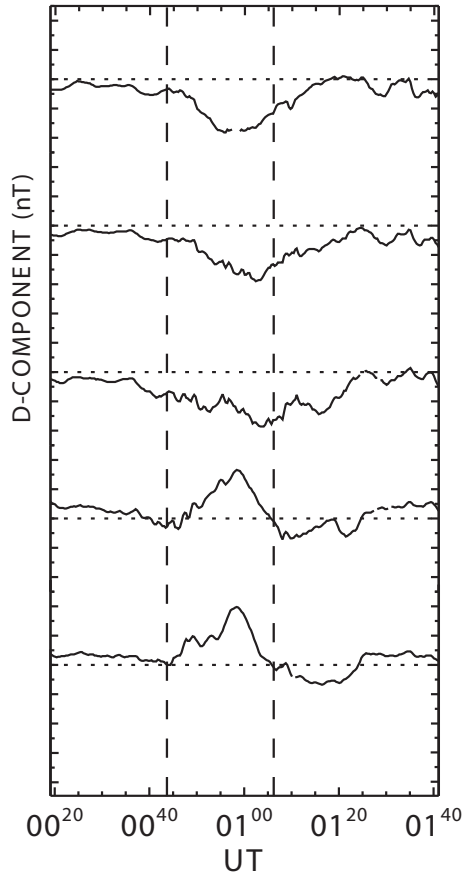

(iii)

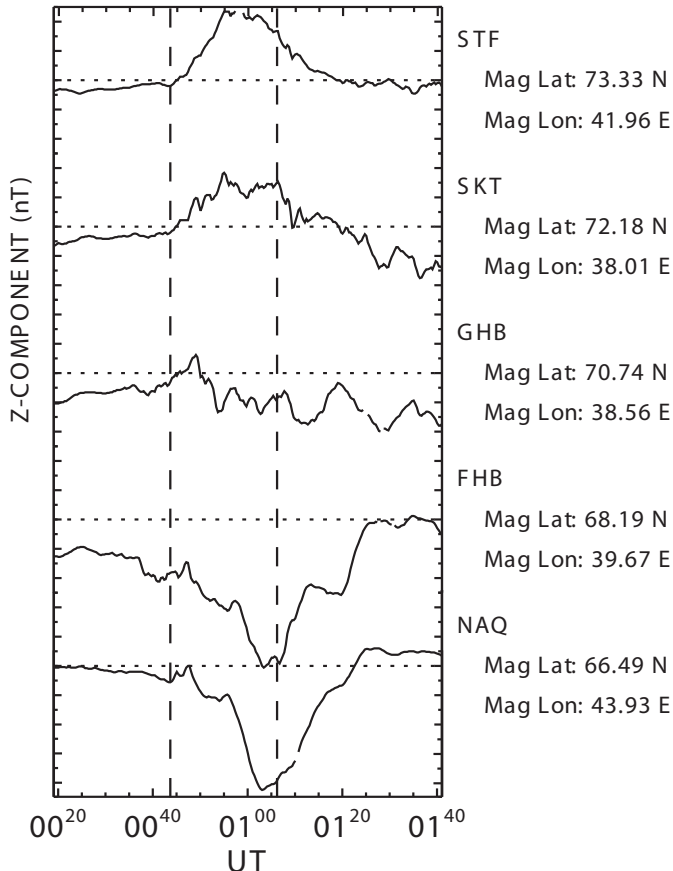

Fig. 3. $H, D$, and $Z$-component magnetic field measurements from the meridional Greenland west coast magnetometer array (see Fig. 1b), for 00:20-01:40 UT on 2 December 1999. The separation between magnetometer baselines (horizontal dotted lines as in Fig. 2b) is $250 \mathrm{nT}$. Vertical dashed lines are as in Fig. 2.

trojet was located near GHB at $\sim 71^{\circ}$. The onset times of the expansion and recovery phases, 00:44 UT and 01:06 UT, respectively, have thus been based on the onset and decay of the negative bay at GHB. Although the expansion onset time is similar at all stations within the electrojet region, GHB is the last station to observe the start of the recovery phase. The location of the center of the disturbance is confirmed by the $Z$ component data displayed in panel (iii), which show nearzero perturbations throughout the interval at station GHB, with negative perturbations at FHB and NAQ in the equatorward region, and positive perturbations at SKT and STF in the poleward region. The negative $Z$ perturbations peak at station FHB, while the positive perturbations peak at STF (and fall to smaller values at GDH at $\sim 76^{\circ}$ and UMQ at $\sim 77^{\circ}$, data not shown). These data thus suggest a westward electrojet spanning $\sim 68^{\circ}-73^{\circ}$, centered near $\sim 71^{\circ}$. The $D$ component data are shown in panel (ii). Relatively strong negative perturbations observed at STF in the poleward region decline with latitude at SKT and GHB, and reverse to positive at FHB and NAQ. These data suggest the presence of additional currents which are directed poleward at GHB, SKT, and SFT, and equatorward at FHB and NAQ.

\subsection{Other substorm indicators}

Figure 4 provides a plot of other parameters which give information on the nature and timing of the disturbance. The first three panels show $H$ component data from the lower- latitude magnetometer stations (green in Fig. 1b) which have been band-pass filtered between 20-200 s to exhibit signals in the Pi2 band. Station NAQ, which is directly equatorward of the main substorm-disturbed region, detects a large amplitude $\mathrm{Pi} 2$ starting at 00:44 UT, confirming our determination of the onset time of substorm expansion. To the east and west, FAR and PBQ both see Pi2s of a smaller amplitude shortly afterwards, indicating the large-scale response to the substorm disturbance.

The lower panels of Fig. 4 show data from geostationary spacecraft. Panels four and five show energetic electron data from LANL-97A and 1994-084, respectively. Both observe relatively weak dispersed injection signatures 10-20 min after substorm onset, consistent with their dawn sector location (see Fig. 1b) (Reeves et al., 1991). No significant ion injections were observed. The bottom three panels show GOES-8 magnetic field data in GSM coordinates. At the time of the substorm, GOES-8 was located at $\sim 20: 00$ MLT, $\sim 1$ h MLT to the west of the disturbed region, as indicated in Fig. $1 \mathrm{~b}$. The dashed curves show model magnetic field values derived from the T-89 $K_{p}=2$ model (Tsyganenko, 1989). Both $B_{x}$ and $B_{y}$ components follow the model field closely, while $B_{z}$ displays a clear increase in magnitude starting at 01:05 UT, which subsequently increases during the recovery phase to values well in excess of the model. This is likely due to the return of magnetic flux from the tail to the inner magnetosphere as a consequence of the substorm. 


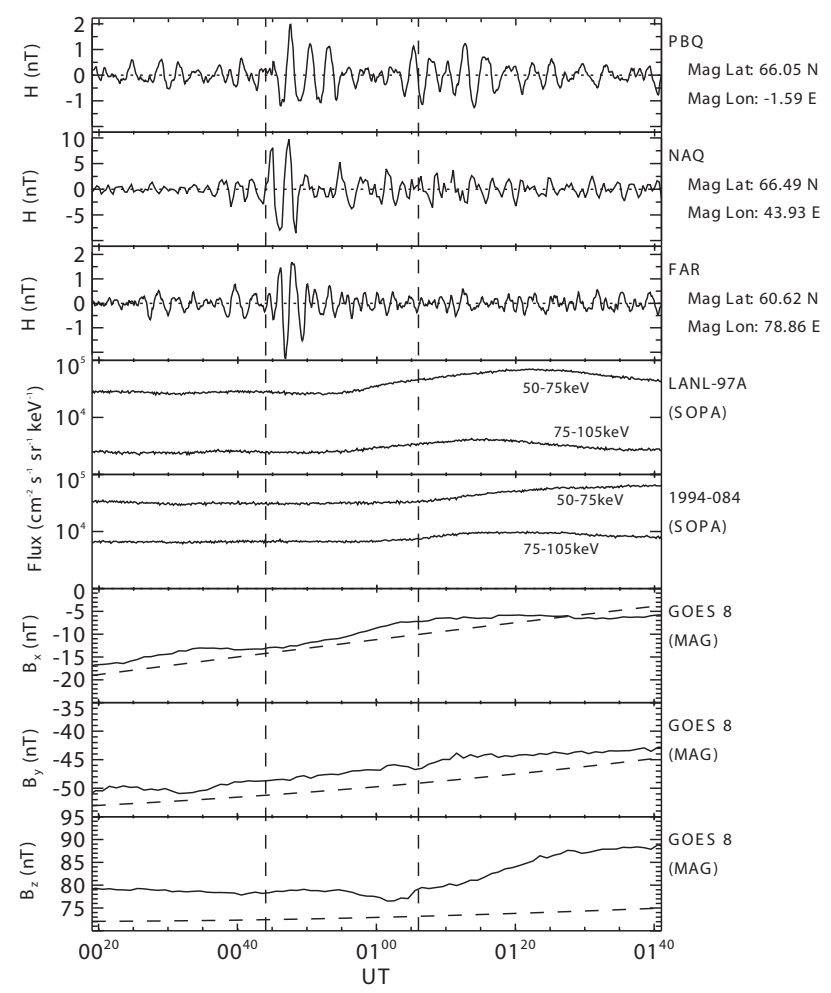

Fig. 4. The upper three panels show filtered (20-200s) Hcomponent magnetic field measurements from the low-latitude magnetometer array (green dots in Fig. 1b) for the interval 00:2001:40 UT on 2 December 1999. Panels three and four similarly show geostationary electron fluxes in two energy channels (50-75 keV and 75-105 keV) from the SOPA instruments on the LANL-97A and 1994-084 spacecraft, while the bottom three panels show geostationary magnetic field measurements from the GOES-8 spacecraft in GSM coordinates. The dashed curves on the GOES-8 plots show the $\mathrm{T}-89$ model magnetic field for $\mathrm{Kp}=2$. The vertical dashed lines are as in Fig. 2.

\subsection{SuperDARN measurements of convection velocities}

We now consider the SuperDARN data, and begin in Fig. 5 by showing line-of-sight velocity data from selected radar beams, the directions of which are indicated by the red lines in Fig. 1a. In the top panel we also show the $H$ component data at GHB, as an indicator of simultaneous magnetic activity. The vertical dashed lines are as in previous figures.

As illustrated in Fig. 1a, the Goose Bay radar observed the pre-midnight substorm sector during the interval of the disturbance. Data from beam 1, shown in the second panel of Fig. 5, clearly indicate a change in both direction and magnitude of the flow shortly after expansion phase onset. Before onset, the radar observed flows of $\sim 100 \mathrm{~m} / \mathrm{s}$ towards the radar (green) above $\sim 72^{\circ}$, reversing to $\sim 500 \mathrm{~m} / \mathrm{s}$ away from the radar (orange) below that latitude. This is indicative of the pre-midnight flow reversal boundary lying within the field-of-view during this time. At the onset of the expansion phase, the poleward region of backscatter abruptly disappeared, indicative of enhanced precipitation within the field-

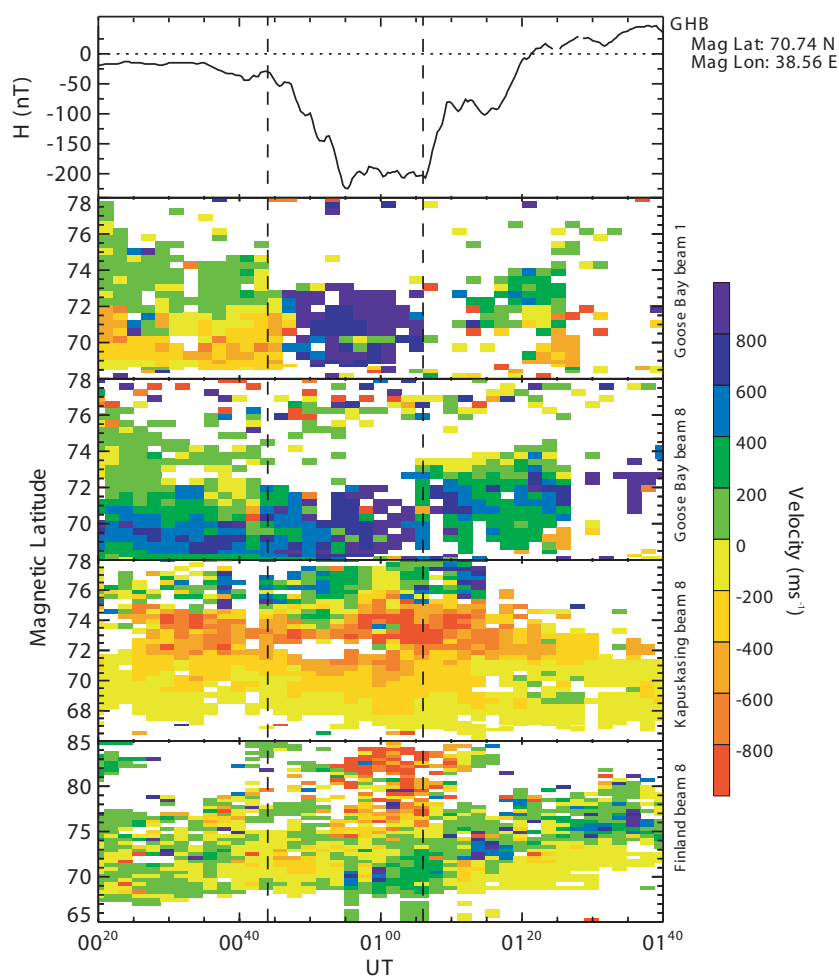

Fig. 5. SuperDARN line-of-sight velocity measurements from the radar beams indicated in Fig. 1a are shown for the interval 00:2001:40 UT on 2 December 1999. In the top panel the $H$-component magnetic field measured by the GHB magnetometer is also shown for purposes of comparison. The vertical dashed lines are as in Fig. 2.

of-view. We mentioned in the Introduction that a reduction in backscatter coverage due to D-region absorption is a common feature of the expansion phase. Fortunately, however, the equatorward region of backscatter remained, and reveals a remarkable change in the flow which developed in concert with the substorm bay at GHB. Within $\sim 4$ min (two radar cycles) of the onset, the flow below $\sim 73^{\circ}$ changed to become strongly equatorward (deep blue and purple) with lineof-sight speeds in excess of $\sim 1000 \mathrm{~m} / \mathrm{s}$. These flows continued during the expansion phase until 01:06 UT, when, in conjunction with the start of the recovery phase, the backscatter coverage became significantly reduced along the whole beam. When partial coverage was resumed, at $\sim 01: 12 \mathrm{UT}$, the line-of-sight flows were much reduced, and a flow reversal boundary was again present within the field-of-view after $\sim 01: 20$ UT.

Related effects can be seen in the data from Goose Bay beam 8, shown in the third panel of Fig. 5. Here the lineof-sight flow is equatorward along almost the whole beam prior to the beginning of the expansion phase. At onset, the backscatter coverage is again reduced in the poleward region. In the equatorward region, the line-of-sight flow intensified over the initial $\sim 6 \mathrm{~min}$ as the bay developed at GHB, and reached $\sim 1000 \mathrm{~m} / \mathrm{s}$ after $\sim 00: 54 \mathrm{UT}$. In this case, however, 

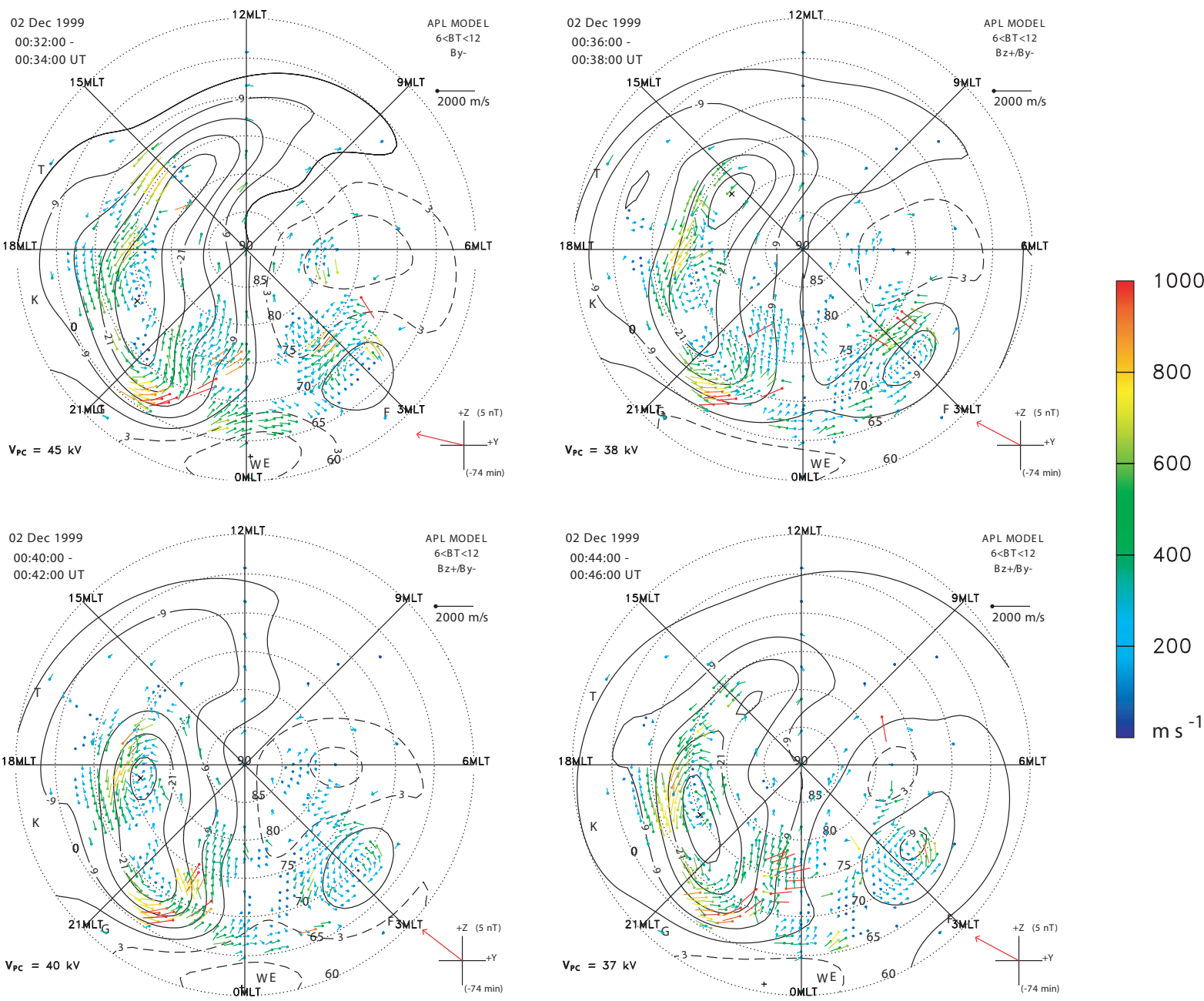

Fig. 6a

Fig. 6. Streamlines and vectors of the ionospheric flow derived from the six-radar SuperDARN velocity measurements are shown on a geomagnetic grid, obtained from the Map-Potential algorithm (see Sect. 2.1). The intervals displayed are (a) 00:32-00:46 UT, (b) 00:4801:02 UT and (c) 01:04-01:18 UT on 2 December 1999. on 2 December 1999. Four maps are shown in each panel (a-c), corresponding to every other 2-min radar experiment cycle. Indicated at the bottom of each map is the total transpolar voltage $V_{P C}$ (left-hand corner), and the direction and magnitude (in the $Y-Z$ plane) of the IMF (right-hand corner). In the top right-hand corner we indicate the flow model employed to stabilize the potential solution in regions where no data are available, obtained from the statistical study of Ruohoniemi and Greenwald (1996).

recovery saw an expansion in the region of backscatter, such that the changes in flow can be followed more fully. The flow remained equatorward along most of the beam, and showed a rapid reduction to smaller values in approximate concert with the decline in the bay amplitude.

Turning to the data from Kapuskasing beam 8 , located in the dusk sector (Fig. 1a) and shown in panel four, it can be seen that prior to onset, the line-of-sight flow below $\sim 75^{\circ}$ is poleward, away from the radar, becoming weaker and directed towards the radar at higher latitudes. Referring to Fig. 1a, it seems evident that these flows relate to the sunward and poleward flows of the normal twin-cell pattern in the dusk sector below $\sim 75^{\circ}$, and to the flow-reversal boundary and polar cap at higher latitudes. After expansion onset a similar pattern remained, but with the flow gradually strengthening over $\sim 10 \mathrm{~min}$ as the substorm developed. It then declined again over $\sim 10 \mathrm{~min}$ after the onset of recovery. Thus, flow effects are also observed in the dusk sector outside the immediate vicinity of the substorm-disturbed region, which we infer to be associated with an enhancement of the twin-cell pattern. It is also notable that no drop-out in backscatter was observed in this sector, but was rather enhanced in this case. Similar comments apply to the dawn sector data from Finland beam 8 (see Fig. 1a), shown in the bottom panel. Here flows are initially weak. However, the expanded coverage available during the expansion phase shows the development of a strong poleward flow away from the radar above $\sim 75^{\circ}$ after $\sim 00: 54 \mathrm{UT}$, which declined over $\sim 10$ min during recovery. We will show below that this flow was also connected to the return flow of a twin-vortex pattern 

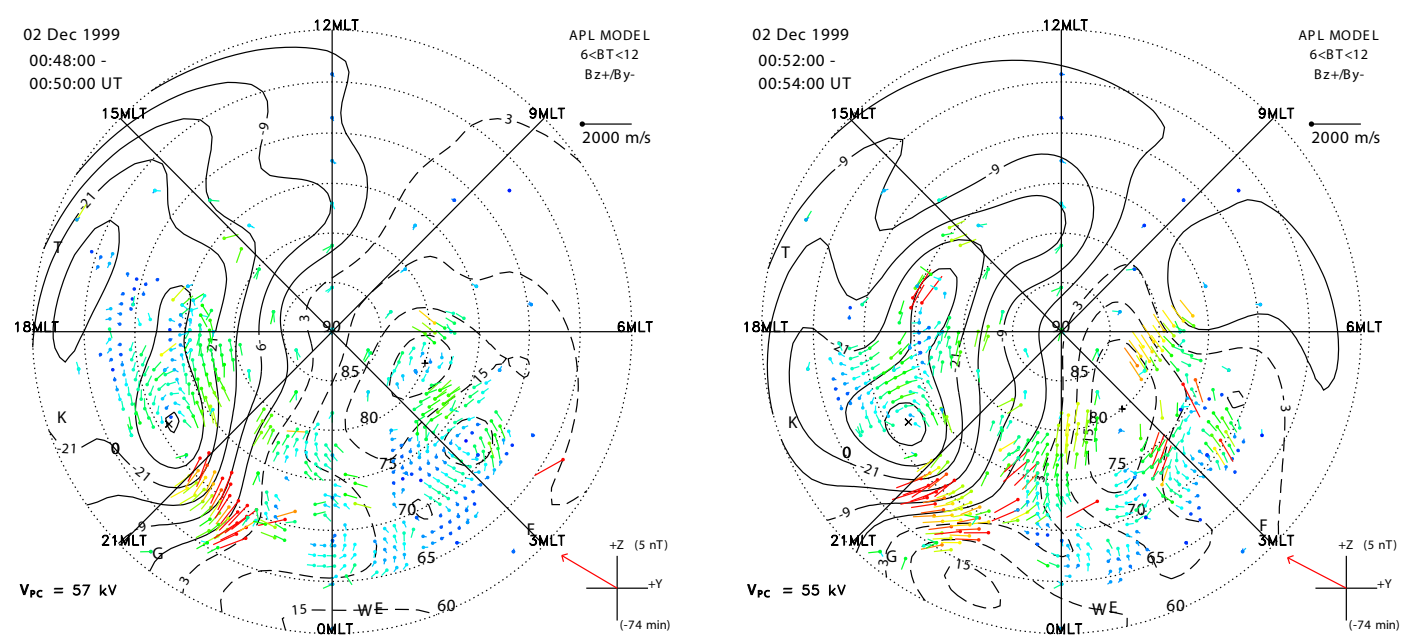

1000

800

600
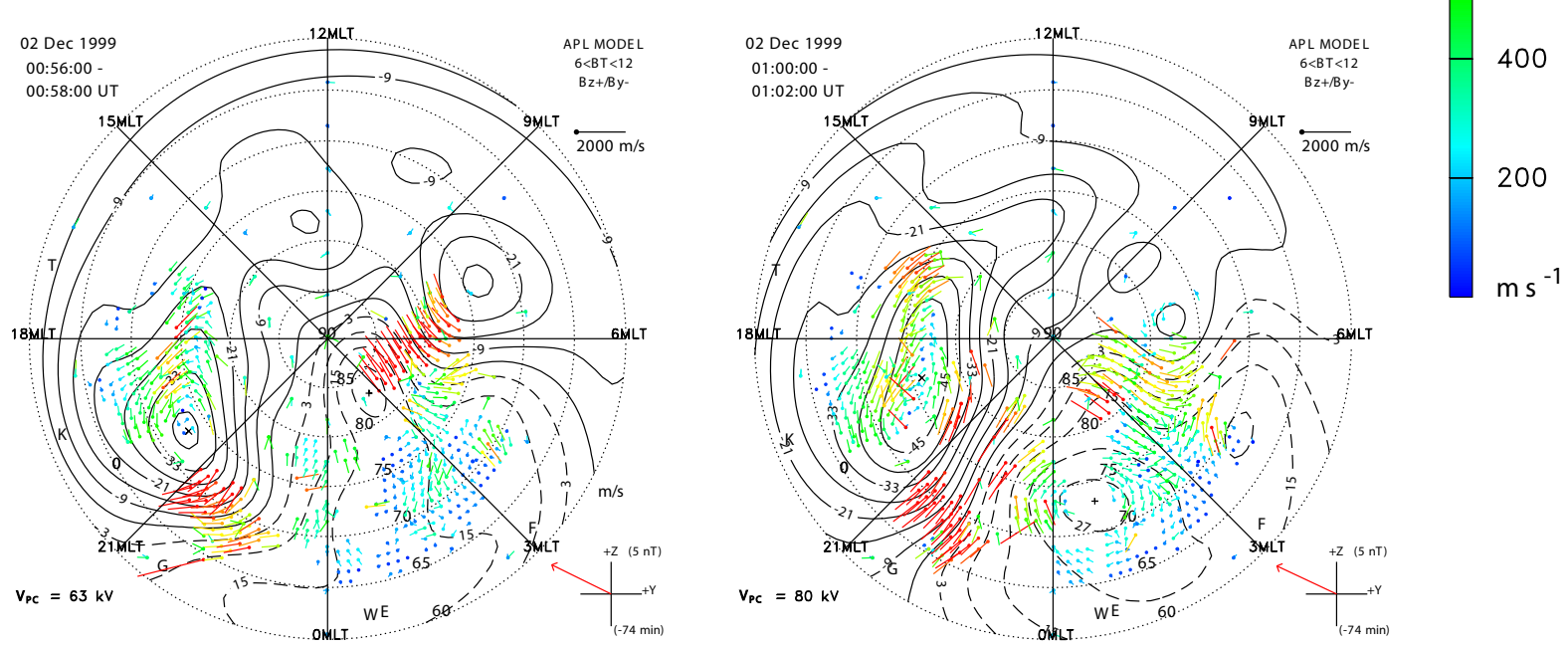

Fig. 6b

Fig. 6. Streamlines and vectors of the ionospheric flow derived from the six-radar SuperDARN velocity measurements are shown on a geomagnetic grid, obtained from the Map-Potential algorithm (see Sect. 2.1). The intervals displayed are (a) 00:32-00:46 UT, (b) 00:4801:02 UT and (c) 01:04-01:18 UT on 2 December 1999. on 2 December 1999. Four maps are shown in each panel (a-c), corresponding to every other 2-min radar experiment cycle. Indicated at the bottom of each map is the total transpolar voltage $V_{P C}$ (left-hand corner), and the direction and magnitude (in the $Y-Z$ plane) of the IMF (right-hand corner). In the top right-hand corner we indicate the flow model employed to stabilize the potential solution in regions where no data are available, obtained from the statistical study of Ruohoniemi and Greenwald (1996).

that is generated and enhanced during the substorm expansion.

In order to visualize the flows from all six radars, Fig. 6 presents convection maps derived using the Map-Potential algorithm discussed in Sect. 2.1. Each map corresponds to one 2-min radar experiment cycle time, and alternate maps are shown over the interval 00:32-01:18 UT spanning the substorm. These are grouped into three sets of four maps each, which correspond to the pre-onset interval, the expansion phase, and recovery. Figure 6a thus displays the convection patterns observed between 00:32 and 00:46 UT, corresponding to the time leading up to and spanning the substorm expansion phase onset at 00:44 UT. During this interval, and for some $\sim 20 \mathrm{~min}$ prior to it, the IMF had been directed consistently northward, with a larger negative $B_{y}$ com- ponent (see Fig. 2a and the icon at the bottom right-hand corner of the flow maps). A reasonably steady and well-defined twin-vortex flow was present during the interval, with a derived transpolar voltage $V_{P C}$ of $\sim 40 \mathrm{kV}$ (given in the bottom left-hand corner of each plot). Since backscatter was present nearly continuously between the extrema in the voltage values (as can be seen from the coverage of the plotted vectors), these voltages probably represent good estimates of the true values. Asymmetries in the flow pattern are evident during the interval, most probably associated with the negative IMF $B_{y}$ conditions prevailing. Examination of the whole data set after $\sim 00: 10$ UT shows that the polar flow was stronger at dawn than at dusk within the more central polar cap, the flow then generally swinging dawnward as the auroral zone is approached on the nightside, and then duskward 

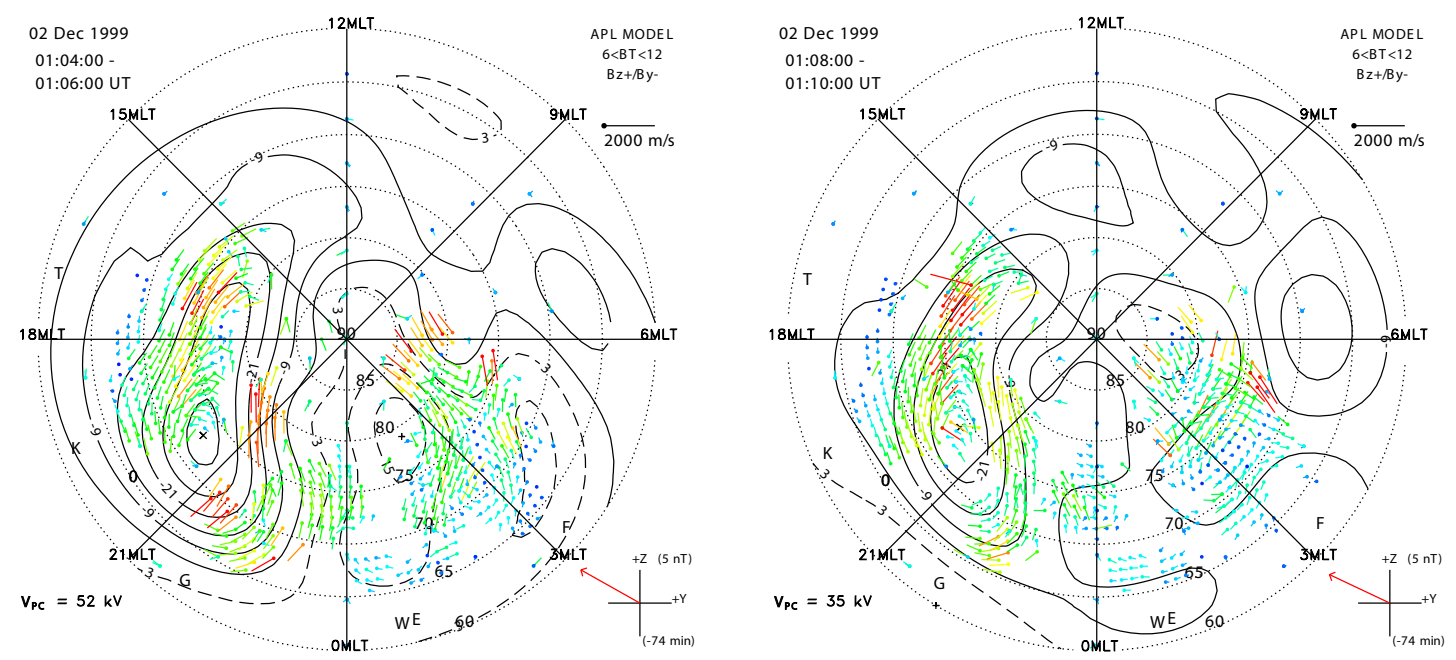

\section{0}
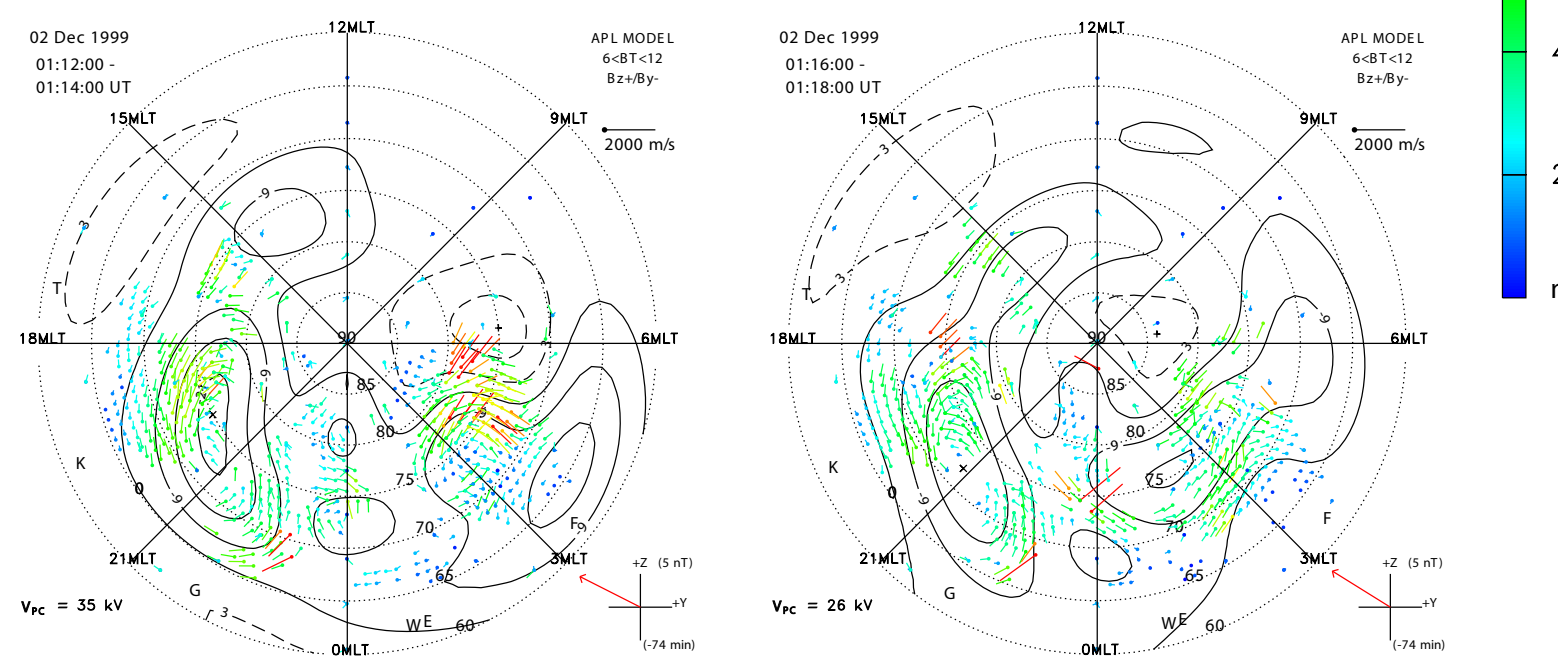

Fig. 6c

Fig. 6. Streamlines and vectors of the ionospheric flow derived from the six-radar SuperDARN velocity measurements are shown on a geomagnetic grid, obtained from the Map-Potential algorithm (see Sect. 2.1). The intervals displayed are (a) 00:32-00:46 UT, (b) 00:4801:02 UT and (c) 01:04-01:18 UT on 2 December 1999. on 2 December 1999. Four maps are shown in each panel (a-c), corresponding to every other 2-min radar experiment cycle. Indicated at the bottom of each map is the total transpolar voltage $V_{P C}$ (left-hand corner), and the direction and magnitude (in the $Y-Z$ plane) of the IMF (right-hand corner). In the top right-hand corner we indicate the flow model employed to stabilize the potential solution in regions where no data are available, obtained from the statistical study of Ruohoniemi and Greenwald (1996).

within the nightside auroral zone, to form a crescent-shaped nightside flow cell (the dusk cell) spanning the midnight sector. During the interval shown in Fig. 6a, however, the flow in the post-midnight tip of the crescent appears to have broken into a separate clockwise vortex (centered at $\sim 70^{\circ}$ latitude and $\sim$ 03:00 MLT), possibly caused by inhomogeneous reconnection rates in the pre-onset tail. The last map in Fig. 6a spans the 2-min interval immediately following the onset of expansion at 00:44 UT. It shows little change compared with the previous three maps, or indeed those of the previous $\sim 20 \mathrm{~min}$.

Figure $6 \mathrm{~b}$ shows the situation between 00:48 and 01:02 UT, corresponding to the expansion phase of the substorm. Here we see the immediate presence of a band of fast equatorward flow in the pre-midnight sector, as noted above in the Goose Bay data. The longitudinal width appears to be $\sim 1.5 \mathrm{~h}$ MLT, from $\sim 21: 00$ to $\sim 22: 30$ MLT, as determined from the available radar coverage. A smaller enhancement of equatorward flow also occurred in the post-midnight sector, together with a distinct zone of avoidance of the flow streamlines (i.e. a region of relative stagnation) in the immediate pre-midnight sector, centered near $\sim 70^{\circ}$. We will show below that this zone corresponds to the brightest region of auroral enhancement during the substorm. Towards dawn and dusk, growing return flows are also seen, associated with the line-of-sight velocity enhancements observed by the Kapuskasing and Finland radars noted above. Overall it is seen that a twin-vortex flow system is excited in the nightside 
ionosphere during the expansion phase, with foci lying at $\sim 20: 00$ MLT in the pre-midnight sector and at $\sim 01: 00$ MLT in the post-midnight sector, at a latitude of $\sim 73^{\circ}$. The overall transpolar voltage associated with the flow grew from $37 \mathrm{kV}$ at onset to peak at $80 \mathrm{kV}$ at 01:00 UT. The excited flows extend into the dawn-dusk and dayside sector, where the flow reversal boundary also appears to undergo a small simultaneous latitudinal contraction. On the dusk meridian, for example, where the boundary is relatively well-defined, a poleward displacement of $\sim 1^{\circ}$ is evident in Fig. $6 \mathrm{~b}$. This motion will be investigated further in Sect. 3.7.

Figure $6 \mathrm{c}$ shows the transition to the recovery phase, between 01:04 and 01:18 UT. In the first map, 01:04-01:06 UT, the equatorward flows in the pre-midnight sector had already decreased significantly, with $V_{P C}$ correspondingly reducing to $57 \mathrm{kV}$. Over the next $\sim 10 \mathrm{~min}$, the flows continued to diminish rapidly, and while remaining essentially similar to those occurring before the substorm, the flows became more structured and variable.

3.6 VIS UV Earth camera auroral images and magnetic disturbance vectors

In this section we now examine how the flows described above relate to the substorm aurora, and to the magnetic disturbance, in so far as the latter can be determined from the ground station coverage. Figure 7 shows auroral images obtained by the VIS Earth camera, on which SuperDARN flow vectors and Map-Potential streamlines have been superposed (black), together with pseudo-flow vectors obtained from ground magnetic observations (red). Each UV image corresponds to a 32.5-s integration, and we show each of the images obtained, at intervals of $\sim 5 \mathrm{~min}$, spanning the substorm. The SuperDARN map corresponds to the 2-min interval into which the center time of the UV image falls. The magnetic data have been averaged over the same 2-min intervals as the SuperDARN scans, and thus correspond similarly to the UV image.

In deriving the magnetic vectors, we have assumed that the perturbations are due to overhead Hall currents, and have thus rotated them anti-clockwise through $90^{\circ}$ to point in the direction of the inferred flow. If this assumption is valid, the vectors should be aligned with the radar vectors, with the ratio of their lengths being determined by the local heightintegrated Hall conductivity. For the scales chosen in the figure, the two vectors would have equal length for a heightintegrated conductivity of $\sim 1.6$ mho (no correction having been made for ground induction effects). In order to obtain useful results, baseline values have to be chosen carefully for each magnetometer station. Examination of the records indicates that after the substorm, the magnetosphere returned to a quiet state during an extended interval of northward IMF (see Fig. 2a). Those stations for which a quiet-time baseline is routinely determined (e.g. the Greenland chain) were all found to have returned close to that baseline by $\sim 04: 00$ UT. Consequently, for simplicity and consistency we have chosen the baseline for all stations as the field measured at 04:00 UT.
This is the baseline used in all plots of unfiltered magnetic data displayed in this paper.

Figure 7a begins by showing two UV images corresponding to the interval before expansion phase onset, centered at 00:33:16 and 00:37:47 UT. The UV auroras form an essentially continuous band circling the pole, reaching $\sim 70^{\circ}$ on the nightside and $\sim 80^{\circ}$ on the dayside, and being wider and reaching to higher latitudes at dawn compared with dusk. The latter asymmetry is likely to be associated with the negative IMF $B_{y}$ conditions prevailing. Peak emission intensities are $\sim 5 \mathrm{kR}$, near to midnight. Comparison with the flow vectors shows that in the region of SuperDARN radar coverage, the auroras lie in a region of westward flow located equatorward of the flow reversal boundary. Due to the (IMF $B_{y}$-related) asymmetry in the flow pattern, this statement applies continuously from the afternoon sector at $\sim 15: 00$ MLT, through dusk and midnight, to the post-midnight sector at $\sim 04: 00$ MLT. Throughout this sector, the poleward boundary of the auroras lies $\sim 1^{\circ}-2^{\circ}$ equatorward of the flow reversal boundary, the latter being a reasonable initial proxy for the open-closed field line boundary. It is also notable that while substantial equatorward-directed flow is clearly present on the nightside during the pre-onset interval, the locations of neither the flow reversal boundary nor the auroras change significantly. If we take the pre-midnight sector, for example, the equatorward flow at $\sim 70^{\circ}$ latitude (near the flow reversal and auroral boundaries) is typically $\sim 300 \mathrm{~m} / \mathrm{s}$. At this speed, the boundaries would move equatorward by $\sim 5^{\circ}$ of latitude in the $\sim 30 \mathrm{~min}$ prior to substorm onset if they moved with the flow (i.e. if the boundaries were adiaroic). This clearly is not the case. Examination of the data from the beginning of the day shows that the boundaries move less than $\sim 1^{\circ}$ during this interval. Consequently, we conclude that the plasma flows through essentially steady-state nightside boundaries during this period. We thus infer that essentially steady nightside reconnection was in progress at a rate of $\sim 40 \mathrm{kV}$ during the pre-onset period, presumably driven by continuous dayside reconnection with the $B_{y}$-dominated IMF. This situation contrasts with the usual growth phase scenario in which the polar cap and auroral zone expand prior to expansion phase onset, as a consequence of unbalanced magnetopause reconnection (McPherron, 1970). Examination of the pseudo-flow magnetic vectors in Fig. 7a shows that they are quite small, but nevertheless often in reasonable agreement with the direction of the SuperDARN flows (though counter-examples are also evident). Comparison of the lengths of the vectors in the auroral region suggests Hall conductivities of $\sim 1-2$ mho.

Figure $7 \mathrm{~b}$ similarly displays two images which span the onset of the expansion phase at 00:44 UT, these being centered at 00:43:41 and 00:48:12 UT, respectively. Comparing these with Fig. 7a shows that the aurora has brightened in the midnight sector to $\sim 15 \mathrm{kR}$ and then to $\sim 25 \mathrm{kR}$, and has also expanded poleward by $\sim 3^{\circ}$ to form a well-defined substorm bulge in the local time sector between $\sim 20: 00$ and $\sim 01: 00$ MLT. The bulge thus sat directly between the foci of the developing nightside twin-vortex flow pattern, though the development of the latter in the morning sector was some- 
02 Dec 1999 00:33:16 UT (0032-0034 UT)

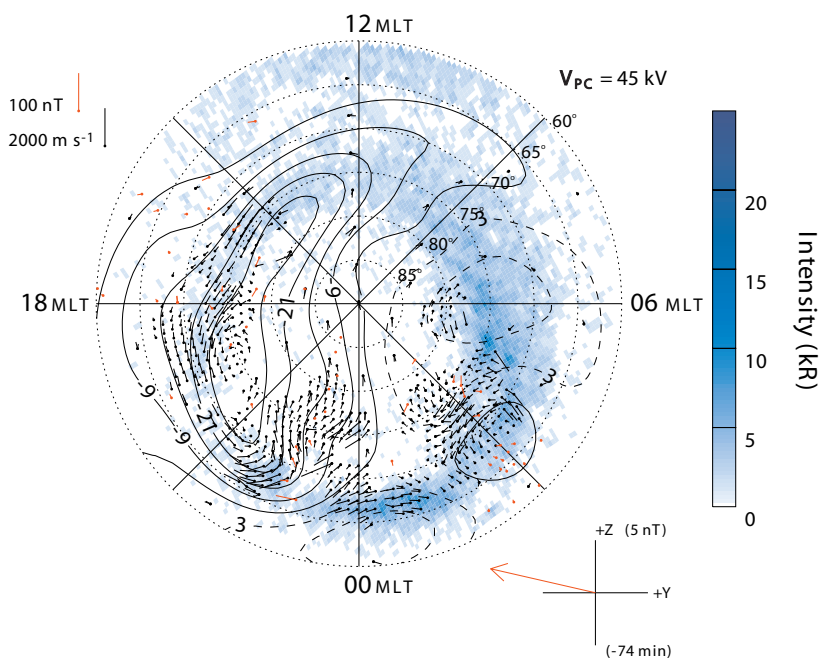

02 Dec $1999 \quad$ 00:37:47 UT $\quad$ (0036-0038 UT)

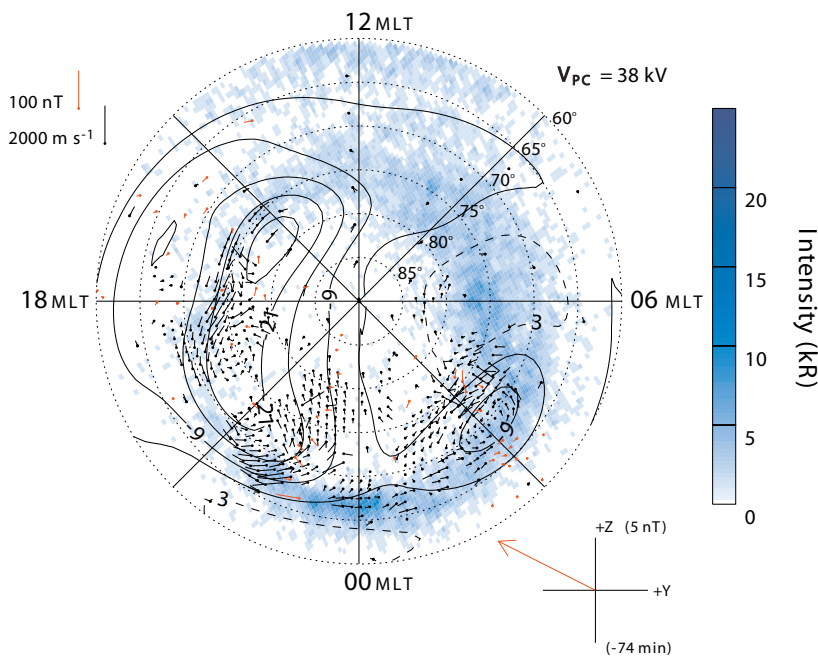

Fig. 7a
Fig. 7. Maps of the UV auroral luminosity projected onto a geomagnetic grid are shown spanning the same interval as in Fig. 6. SuperDARN MapPotential streamlines and flow vectors are superposed. Magnetic data, shown in red, are horizontal magnetic perturbation vectors which have been rotated anti-clockwise through $90^{\circ}$ to point in the direction of the $\boldsymbol{E} \times \boldsymbol{B}$ drift, assuming they are due wholly to overhead Hall currents. Also indicated on the maps is the derived total transpolar voltage and the direction and magnitude (in the $Y-Z$ plane) of the IMF, as in Fig. 6. The time indicated on each plot corresponds to the center time of the $32.5 \mathrm{~s}$ integration interval of the UV image. The superposed SuperDARN and 2-min averaged magnetic vectors correspond to the interval shown in brackets, which encompasses the center time of the UV image. what obscured by the continuing clockwise vortex. The rapid poleward motion of the auroras, taking place in concert with strong and increasing equatorward flow, argues for the occurrence of rapid tail reconnection during this interval. Another feature of note is the zone of flow avoidance mentioned above, which developed in the flow pattern at $\sim 00: 48 \mathrm{UT}$, and was centered near $\sim 70^{\circ}$ in the immediate pre-midnight sector. This zone clearly relates to the blob of brightest auroral emission which lay between $\sim 65^{\circ}$ and $\sim 70^{\circ}$ in this sector. We infer that the flow was diverted around this region due to the high electrical conductivity resulting from the precipitation. Similar effects have been found in previous studies by Kirkwood et al. (1988), Morelli et al. (1995), Yeoman et al. (2000), and Khan et al. (2001). Looking now at the magnetic observations, and identifying the west coast Greenland stations which lie within the meridian of the substorm bulge (see Fig. 1b), it can be seen that the peak disturbance occurred (at station GHB) in the weak-flow region at the poleward border of the auroral emission. The relative lengths of the magnetic and flow vectors in this region indicate Hall conductivities of $\sim 10-20$ mho. It can also be seen from the magnetic vectors that the westward electrojet spanned $\sim 70^{\circ}-73^{\circ}$ at this time (stations GHB to STF on the Greenland west coast chain, see Fig. 3a), and thus spanned, and extended poleward of, the poleward border of the UV emission. Significant magnetic effects were also observed at station IQA lying just poleward of the emission at $\sim 21: 00$ MLT (see Figs. 1b and 2b). No magnetic vectors are shown directly within the blob of strongest UV emission. The only station lying directly within this region was Greenland east station AMK (see Fig. 1b), which observed the largest negative $H$ perturbation globally during the substorm, as previously shown in Fig. 2b. Unfortunately, however, the total horizontal disturbance at AMK cannot be shown because the $D$ component is unavailable 


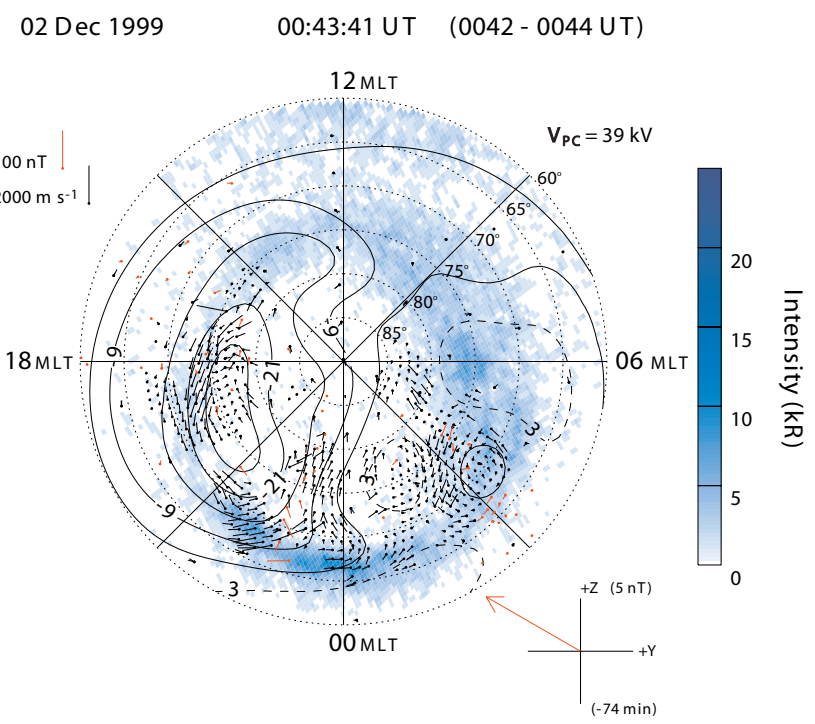

02 Dec 1999 00:48:12 UT $\quad(0048-0050$ UT)

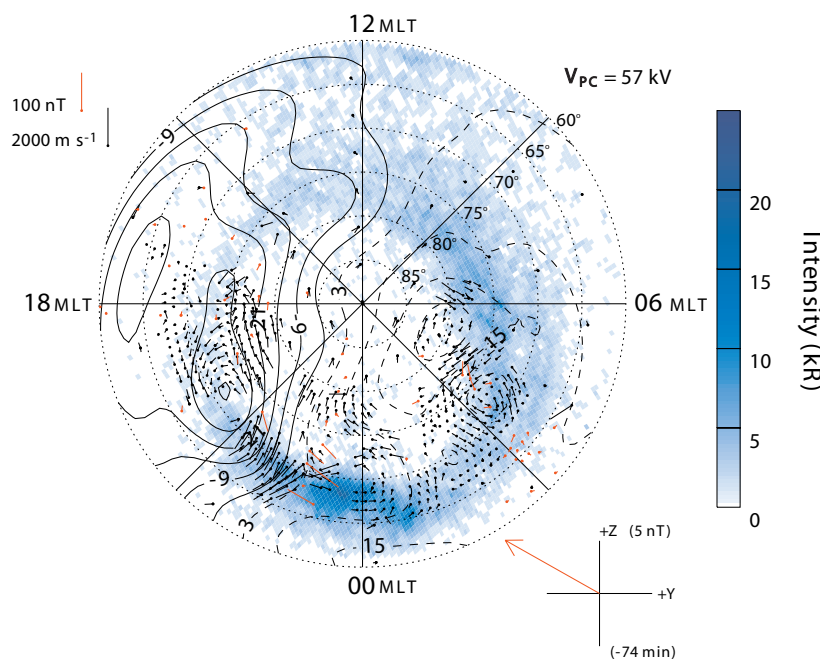

Fig. 7b
Fig. 7. Maps of the UV auroral luminosity projected onto a geomagnetic grid are shown spanning the same interval as in Fig. 6. SuperDARN MapPotential streamlines and flow vectors are superposed. Magnetic data, shown in red, are horizontal magnetic perturbation vectors which have been rotated anti-clockwise through $90^{\circ}$ to point in the direction of the $\boldsymbol{E} \times \boldsymbol{B} \mathrm{drift}$, assuming they are due wholly to overhead Hall currents. Also indicated on the maps is the derived total transpolar voltage and the direction and magnitude (in the $Y-Z$ plane) of the IMF, as in Fig. 6. The time indicated on each plot corresponds to the center time of the $32.5 \mathrm{~s}$ integration interval of the UV image. The superposed SuperDARN and 2-min averaged magnetic vectors correspond to the interval shown in brackets, which encompasses the center time of the UV image.

for this station for this interval.

Figure 7c shows auroral images centered at 00:54:06 and 00:58:37 UT, which span the interval of maximum observed magnetic disturbance at $\sim 00: 55$ UT. Here we see that the substorm auroral bulge had also reached its maximal extent, with the westward electrojet continuing to span and extend poleward of its poleward boundary. Unfortunately there are now no radar flow vectors within the electrojet region that can be compared with the magnetic vectors, and from which conductivity estimates can be made. The flow excited during the substorm is well-established, with $V_{P C} \sim 70 \mathrm{kV}$. Within the bulge, the zone of avoidance near midnight is still evident. Outside the bulge, the enhanced return flows are seen to extend via dusk and dawn into the dayside Hemisphere. Associated magnetic disturbances of modest amplitude are thus produced in the surrounding regions where the conductivity is sufficient to produce a measurable effect.

Figure $7 \mathrm{~d}$ shows images, centered at 01:04:32 and 01:09:02 UT, which span the start of the substorm recovery phase at $\sim 01: 06$ UT. During this interval the peak auroral intensities within the bulge had diminished significantly to $\sim 5 \mathrm{kR}$, similar to values occurring before onset. However, a comparison of the spatial distribution of the aurora with that shown in Fig. 7a shows that a small general poleward displacement of the poleward border of the aurora had occurred, by $\sim 1^{\circ}-2^{\circ}$, even on the dayside. This displacement mirrors the $\sim 1^{\circ}$ poleward motion of the flow reversal boundary at dusk noted above. The magnetic disturbance produced by the westward electrojet at the poleward border was still large, but diminishing, while the magnetic disturbance in the dawn sector had grown to become comparable in intensity. The transpolar voltage associated with the flow also diminished over this interval to pre-onset values.

Figure 7e finally shows two recovery phase images, cen- 
02 Dec 1999 00:54:06 UT (0054-0056 UT)

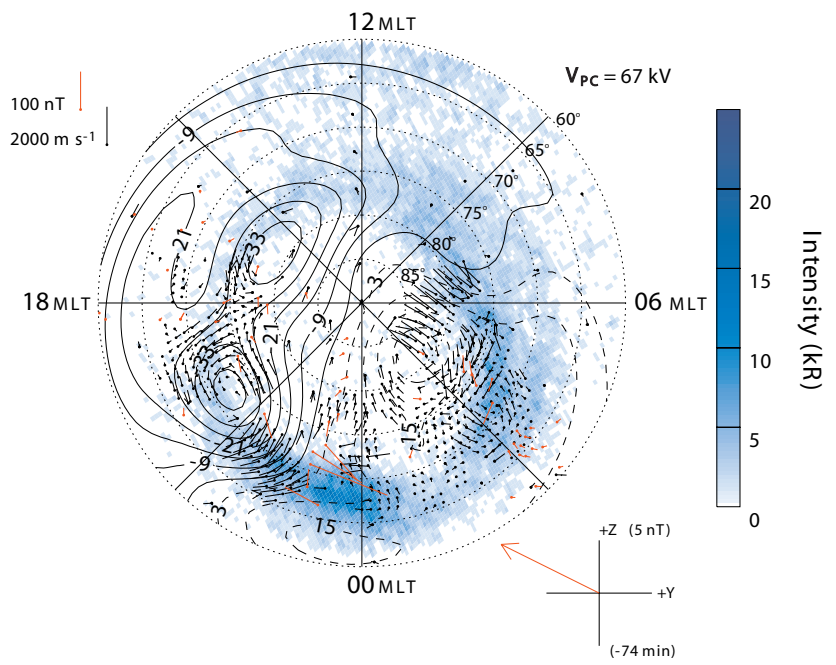

02 Dec 1999

00:58:37 UT $\quad(0058-0100$ UT $)$

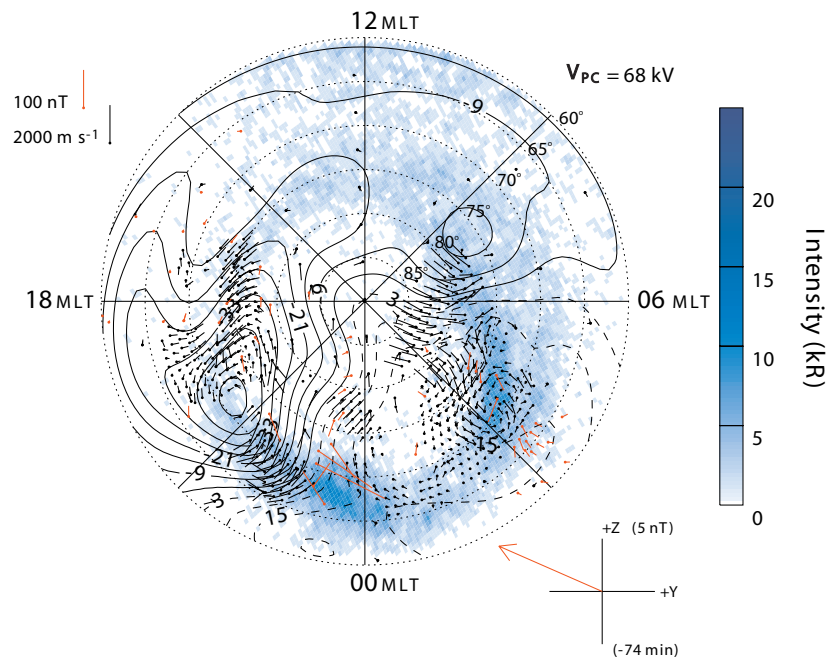

Fig. 7c
Fig. 7. Maps of the UV auroral luminosity projected onto a geomagnetic grid are shown spanning the same interval as in Fig. 6. SuperDARN MapPotential streamlines and flow vectors are superposed. Magnetic data, shown in red, are horizontal magnetic perturbation vectors which have been rotated anti-clockwise through $90^{\circ}$ to point in the direction of the $\boldsymbol{E} \times \boldsymbol{B} \mathrm{drift}$, assuming they are due wholly to overhead Hall currents. Also indicated on the maps is the derived total transpolar voltage and the direction and magnitude (in the $Y-Z$ plane) of the IMF, as in Fig. 6. The time indicated on each plot corresponds to the center time of the $32.5 \mathrm{~s}$ integration interval of the UV image. The superposed SuperDARN and 2-min averaged magnetic vectors correspond to the interval shown in brackets, which encompasses the center time of the UV image. tered at 01:14:57 and 01:19:27 UT. Here the intensity of the bulge had diminished still further, as had the currents and flows. Nonetheless, equatorward flow persisted across the poleward boundary of the auroras in the pre-midnight sector, suggestive of continuing reconnection in the pre-midnight tail. The transpolar voltage values were typically $\sim 35 \mathrm{kV}$, a little less than those occurring before the substorm.

\subsection{Summary of observations}

Figure 8 presents a summary of our main observations, from which we draw the simple, yet unequivocal conclusion that twin-vortex flow was excited in the nightside high-latitude ionosphere by an isolated substorm. The top three panels remind us of the nature of the IMF (lagged by $74 \mathrm{~min}$ ) during the substorm, with $B_{x}$ small and negative, $B_{y}$ large and negative, and $B_{z}$ intermediate in strength and positive. Such interplanetary conditions would be expected to result in mod- est steady convection velocities in the ionosphere, resulting from reconnection with the $B_{y}$-dominated IMF. In fact, under these conditions, the Ruohoniemi and Greenwald (1996) statistical model predicts a transpolar voltage of $33 \mathrm{kV}$. Flows associated with transpolar voltages up to $\sim 80 \mathrm{kV}$, as observed, cannot reasonably be accounted for on this basis. In addition, there are no indications in the dayside auroral data of major magnetopause dynamics during the interval. The fourth and fifth panel summarises the ground magnetic data, emphasising that the disturbance studied has the typical characteristics of an isolated substorm of modest duration and amplitude. Overall, the bay lasted for $\sim 25 \mathrm{~min}$, had a peak amplitude of $\sim 250 \mathrm{nT}$ in the $H$ component, and was preceded by a well-defined Pi2 signal. Weak energetic particle injections and field strength increases were also observed at geostationary orbit, as shown above. The sixth panel in Fig. 8 shows the total transpolar voltage. It seems obvious 


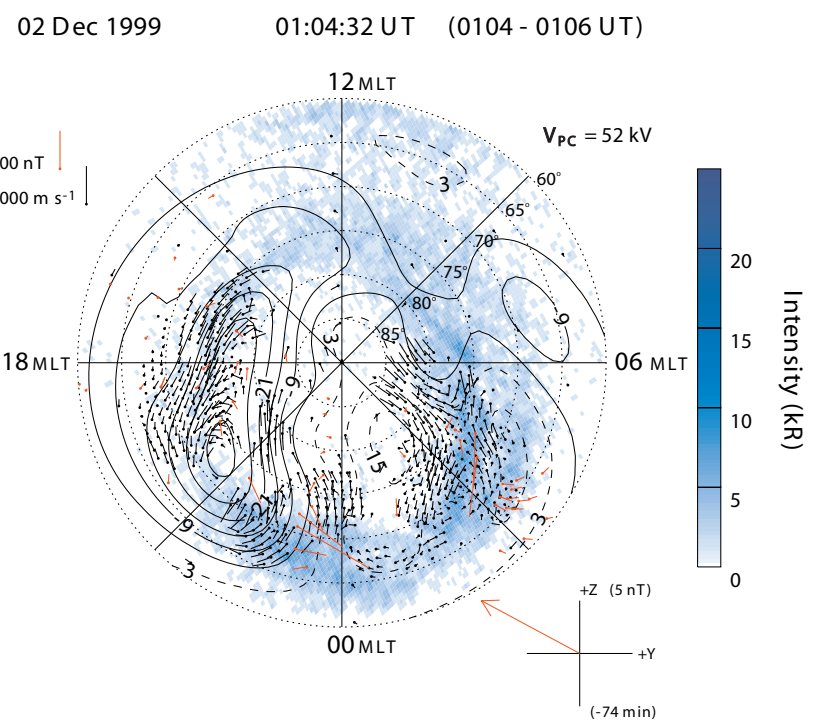

02 Dec 1999 01:09:02 UT (0108-0110 UT)

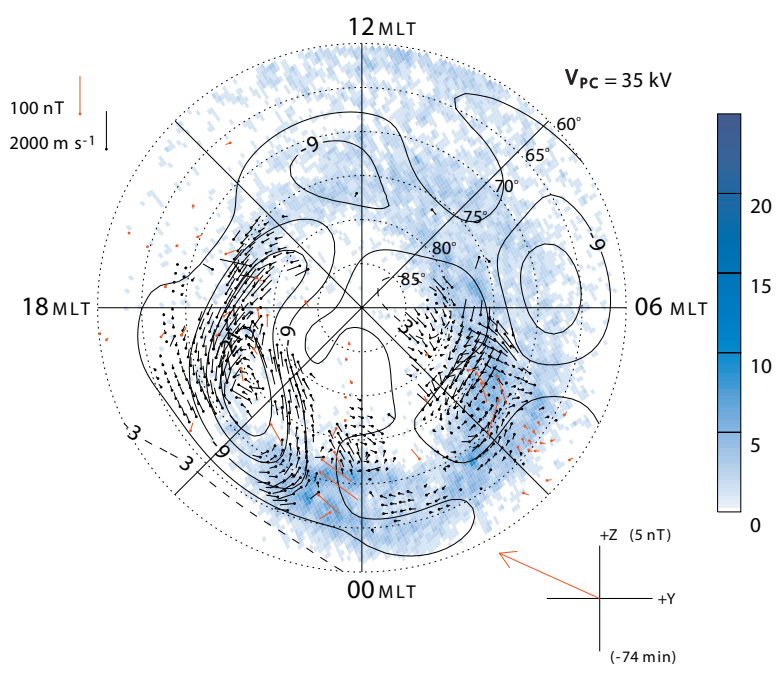

Fig. 7. Maps of the UV auroral luminosity projected onto a geomagnetic grid are shown spanning the same interval as in Fig. 6. SuperDARN MapPotential streamlines and flow vectors are superposed. Magnetic data, shown in red, are horizontal magnetic perturbation vectors which have been rotated anti-clockwise through $90^{\circ}$ to point in the direction of the $\boldsymbol{E} \times \boldsymbol{B}$ drift, assuming they are due wholly to overhead Hall currents. Also indicated on the maps is the derived total transpolar voltage and the direction and magnitude (in the $Y-Z$ plane) of the IMF, as in Fig. 6. The time indicated on each plot corresponds to the center time of the $32.5 \mathrm{~s}$ integration interval of the UV image. The superposed SuperDARN and 2-min averaged magnetic vectors correspond to the interval shown in brackets, which encompasses the center time of the UV image.
Fig. 7d that the excitation of flow relates directly to the interval of substorm expansion, both in terms of the times of increase and decay. Finally, the seventh panel shows the latitude of the flow reversal boundary on the dusk meridian, where it is well-defined, determined from the minimum in the voltage of the Map-Potential fit. Since the solution for the potential is obtained on a discrete latitudinal grid of $1^{\circ}$ resolution, these data are rather marginal for the present purpose. However, as indicated above, they suggest that a small poleward displacement of the boundary took place during the substorm. Averaged over the $\sim 20$-min intervals before and after the substorm, indicated by the horizontal dashed lines, the boundary moved from $\sim 77^{\circ}$ before to $\sim 78^{\circ}$ after, i.e. a displacement of $\sim 1^{\circ}$. The poleward motion of the flow reversal boundary is also evident in the line-of-sight data from beam 8 of the Kapuskasing radar shown in Fig. 5, panel four, although the latitude of the boundary is somewhat lower due to the later MLT.

A few further comments are in order concerning the $V_{P C}$ estimates shown in Fig. 8 (and in Figs. 6 and 7). The first concerns the sensitivity of the results to the statistical flow model employed to stabilize the spherical harmonic solutions in regions where radar data are sparse, as mentioned in Sect. 2.1. This question has been examined quantitatively by re-deriving the voltage estimates using different fixed Ruohoniemi and Greenwald (1996) models appropriate to various IMF conditions. The voltages derived typically vary by only a few $\mathrm{kV}$ from those shown in Fig. 8, such that it is clear that our results depend principally on the radar data, and not upon the statistical model. This may be expected from the fact that our radar data, in general, adequately span the region between the foci of the flow cells, as mentioned above. The importance of continuity of radar data coverage to the voltage estimates is emphasized by the results of a second 
02 Dec 1999 01:14:57 UT (0114-0116 UT)

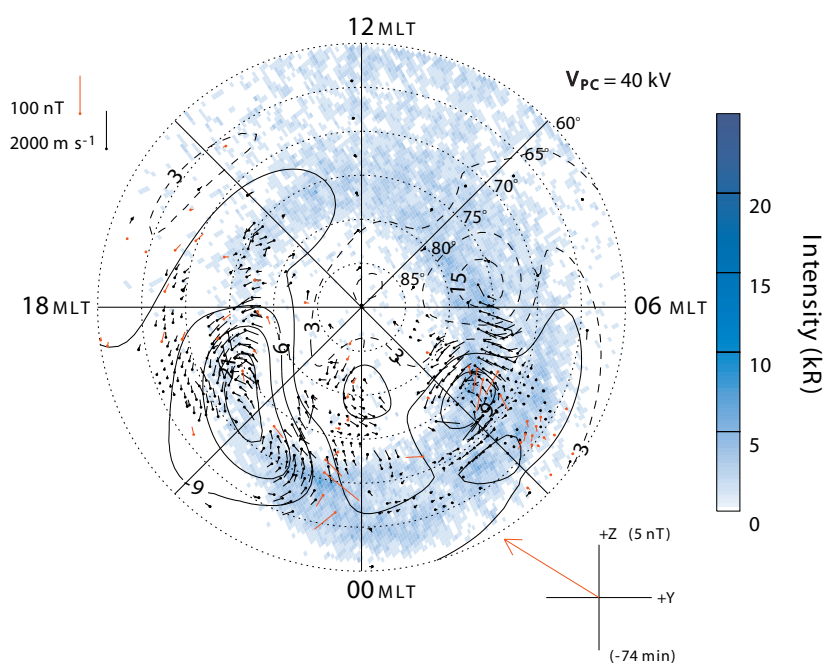

02 Dec 1999 01:19:27 UT $\quad(0118-0120$ UT $)$

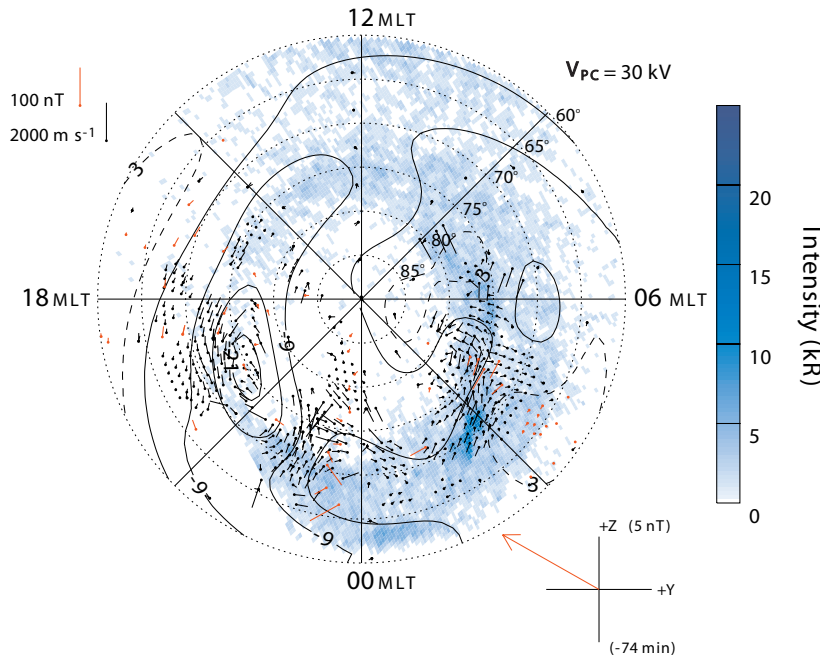

Fig. 7e
Fig. 7. Maps of the UV auroral luminosity projected onto a geomagnetic grid are shown spanning the same interval as in Fig. 6. SuperDARN MapPotential streamlines and flow vectors are superposed. Magnetic data, shown in red, are horizontal magnetic perturbation vectors which have been rotated anti-clockwise through $90^{\circ}$ to point in the direction of the $\boldsymbol{E} \times \boldsymbol{B}$ drift, assuming they are due wholly to overhead Hall currents. Also indicated on the maps is the derived total transpolar voltage and the direction and magnitude (in the $Y-Z$ plane) of the IMF, as in Fig. 6. The time indicated on each plot corresponds to the center time of the $32.5 \mathrm{~s}$ integration interval of the UV image. The superposed SuperDARN and 2-min averaged magnetic vectors correspond to the interval shown in brackets, which encompasses the center time of the UV image. investigation, in which the radar data that show the equatorward surge in the substorm sector (specifically, the Goose Bay data) have been removed prior to employing the MapPotential algorithm. In this case the voltage determinations before and after the substorm are at similar levels to those displayed in Fig. 8. However, during the substorm itself, the voltage peak seen in Fig. 8 is missing entirely. Good continuity of radar data coverage across the substorm-disturbed region is thus essential for adequate estimation of the voltages associated with the flow and their variation during the substorm.

\section{Discussion}

We now discuss the physical origins of the flow excitation, and, following the discussion in the Introduction and that of Siscoe and Huang (1985), Cowley and Lockwood (1992), and Cowley et al. (1992, 1998), we presume that it was due principally to an interval of enhanced reconnection and net open flux closure in the tail. Here we will make estimates of the reconnection rates involved, and of the net flux closure, on this basis.

We pointed out above that in the period prior to expansion onset the steady nature of the flow and precipitation boundaries indicates that this was an interval of quasi-steady balanced dayside and nightside reconnection. In this case, the mean reconnection rates are approximately equal to the polar cap voltage, so that averaging the SuperDARN voltage data over the 20-min interval prior to expansion onset at 00:44 UT yields estimates of the pre-onset reconnection rates (dayside and tail) of $43 \pm 4 \mathrm{kV}$ (mean and standard deviation). In the interval after the substorm, specifically over the period 01:08-01:28 UT, we similarly find that the average polar cap voltage was $33 \pm 5 \mathrm{kV}$. These values indicate 


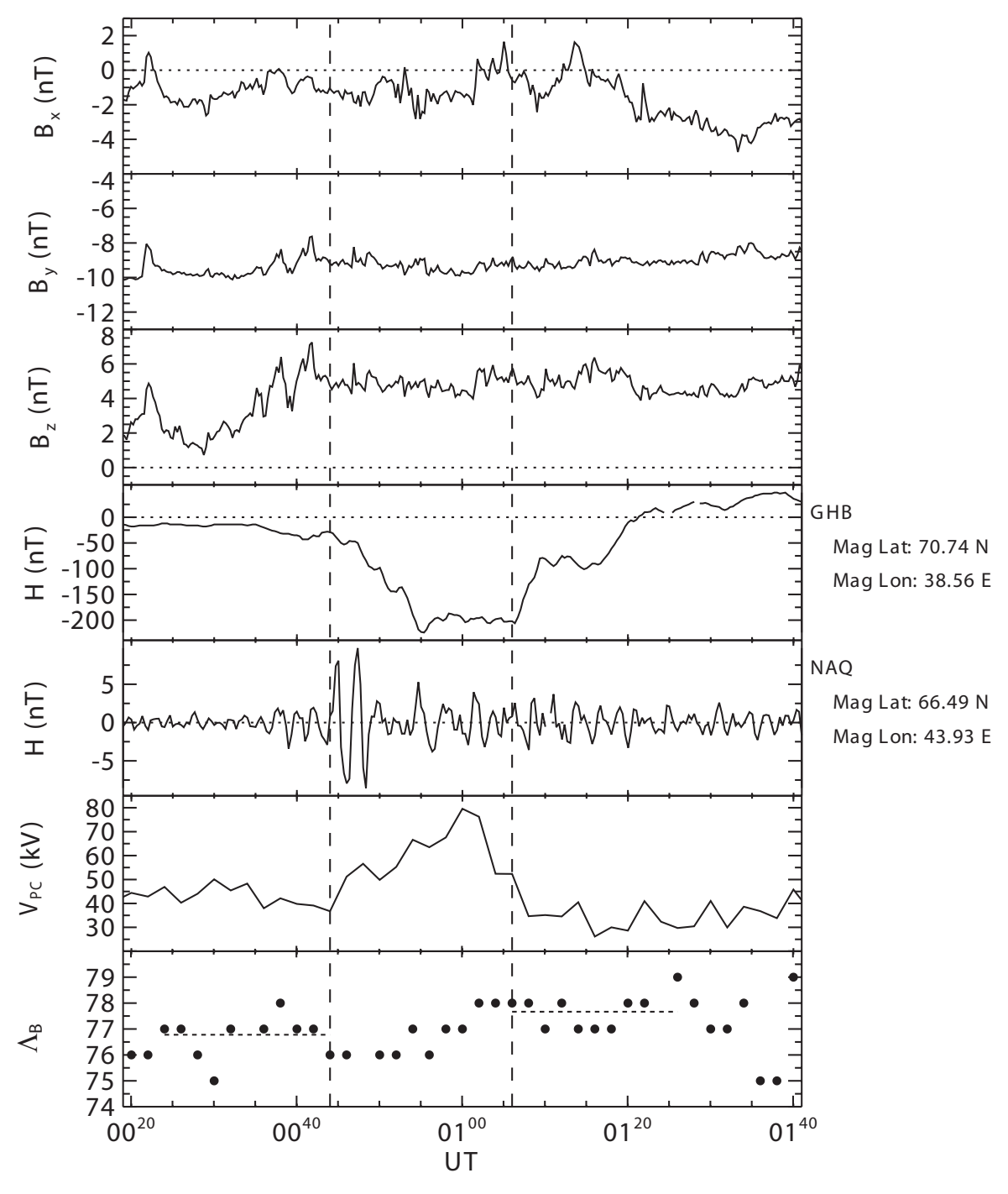

Fig. 8. Summary plot of the principal observed substorm features. The top three panels show the IMF measurements by the ACE spacecraft in GSM coordinates, lagged by $74 \mathrm{~min}$ as in Fig. 2a, shown for the interval 00:20-01:40 UT on 2 December 1999. The fourth panel similarly shows the $H$-component of the magnetic field measured by the GHB magnetometer, while the fifth panel shows the $H$ component magnetic field band-pass filtered between 20 and 200 s measured by the NAQ magnetometer. The sixth panel shows the total transpolar voltage $V_{P C}$ derived from SuperDARN radar data using the Map-Potential algorithm, while the seventh panel shows an estimate of the latitude of the flow reversal boundary on the dusk meridian, obtained from the minimum in the electrostatic potential. The horizontal dashed lines show the location averaged over 20-min intervals before and after the substorm. The vertical dashed lines are as in Fig. 2. that the system was being driven by quasi-steady dayside reconnection at a mean rate between 30 and $45 \mathrm{kV}$ before and after the substorm, and that this was matched by tail reconnection at similar mean rates during these periods. During the substorm, however, we suggest that the tail reconnection rate was temporarily elevated above such values, while it seems most reasonable to suppose that dayside reconnection continued at similar rates of $30-45 \mathrm{kV}$. In support of the latter assumption, we note that the interplanetary conditions, which are primarily deterministic of the dayside reconnection rate, did not change significantly over the interval (e.g. Fig. 8), nor is there any evidence of major dayside dynamics in the auroral data (Fig. 7). If this is correct, we then expect net destruction of open flux to have taken place during the substorm, associated with a contraction of the open-closed field line boundary. Evidence for such contraction, though incomplete, is indeed present in our data. Specifically, we have previously noted that the flow reversal boundary in the dusk sector, where it is most sharply defined, underwent a poleward displacement of $\sim 1^{\circ}$ (Figs. 5-8), near the limit of resolution of the SuperDARN data. It is also evident that a poleward contraction of $\sim 1^{\circ}-2^{\circ}$ took place in the poleward boundary of the UV auroras at most local times (Fig. 7), though the relationship to the motion of the open-closed field line boundary remains somewhat uncertain. Thus, while evidence for contraction exists, its value (of the order of $\sim 1^{\circ}$ ) is imprecisely defined by the data we have at our disposal. The analysis presented below is tailored to accommodate the imprecision in this parameter.

The physical scenario on which the calculation is based is illustrated in Figs. 9 and 10, where we show only the principal features, and not the details specific to the interval considered here, such as the IMF $B_{y}$-related asymmetries in the flow, or the details of the flow suppression effects. In order to set the theoretical scene, we first consider the system response to a short-lived pulse of tail reconnection, shown in Fig. 9. In this diagram the instantaneous open-closed field line boundary is shown by the heavy solid line, while the 


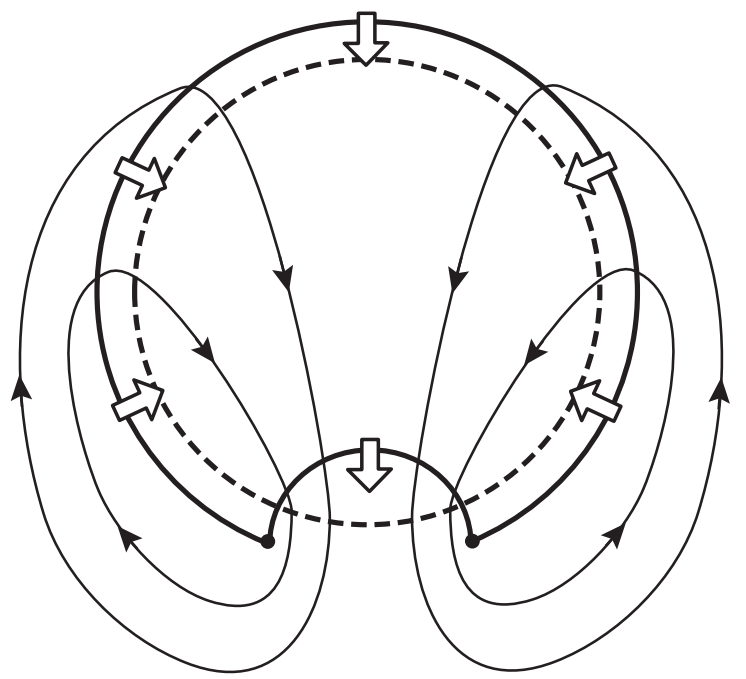

Fig. 9. Schematic of the flow which is excited by a single shortlived pulse of nightside reconnection. The instantaneous openclosed field line boundary is shown by the heavy solid line, while the plasma streamlines are shown by the lighter arrowed lines. The implied boundary motion is illustrated by the large arrows, and the equilibrium boundary position is indicated by the dashed circle.

plasma streamlines are shown by the lighter arrowed lines. We have assumed for simplicity that the initial equilibrium open-closed field line boundary is circular in shape, and that a pulse of tail reconnection has caused a poleward displacement of the boundary in the nightside sector. The flux tubes immediately equatorward of the bulge have thus been newly reconnected in the tail, and have been added to the closed flux tube region in the nightside magnetosphere. This perturbs the system from equilibrium, and excites flow which transports flux from the nightside, around the Earth and towards the dayside, thus forming part of the Dungey cycle. In the ionosphere, this transport is manifest as a twin-vortex flow, which is such that the open-closed field line boundary is carried equatorward in the region of the nightside bulge, and poleward elsewhere. These motions thus return the boundary towards an equilibrium circular shape with a reduced amount of open flux present, shown by the dashed circular line, after which the flow dies away. In effect, the action of the flow is to distribute the newly closed flux in the system, approximately uniformly around the open-closed field line boundary.

The more general situation is illustrated in Fig. 10, where we show the effect of a more extended interval of enhanced tail reconnection that takes place in the presence of steady dayside reconnection, such as we have inferred here. Figure 10a shows the situation in the interval of quasi-steady flows that occurred before substorm expansion onset. The heavy line represents the open-closed field line boundary, with the dot-dashed portion being the merging gap mapping to the nightside reconnection region. The lighter arrowed lines represent the plasma streamlines, which thus cross the boundary only in the region of the merging gap, while being (a)

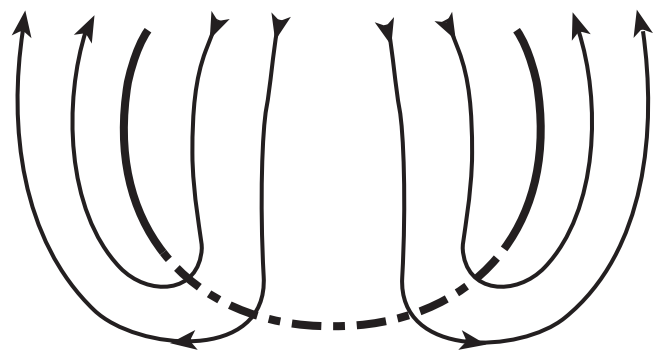

(b)

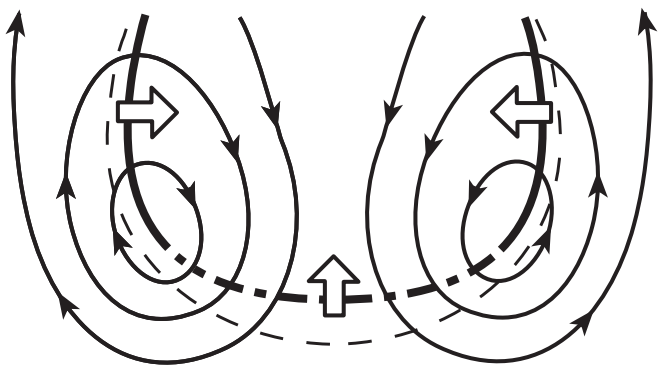

(c)

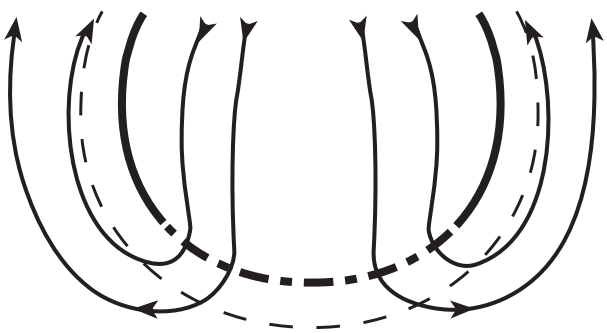

Fig. 10. Sketch showing idealized boundary motions and plasma streamlines (a) before, (b) during, and (c) after the substorm studied here, such that (a) and (c) correspond to quasi-steady states, while (b) corresponds to an interval in which the tail reconnection rate is enhanced and exceeds the dayside rate. The heavy lines show the position of the open-closed field line boundary, which is shown solid in the adiarioc portions and dot-dashed in the region of the nightside merging gap. The large arrows in (b) show the motion of the boundary after the enhancement of tail reconnection rate has taken place, while the dotted line in (b) and (c) shows the initial position of this boundary for ease of comparison. The lighter arrowed lines show the plasma streamlines.

aligned with the boundary in the adiaroic regions outside. Figure $10 \mathrm{~b}$ corresponds to the expansion phase of the substorm, in which tail reconnection closes open flux at an enhanced rate (larger than the dayside reconnection rate), such that the open-closed field line boundary now moves poleward as shown, beginning in the sector of the merging gap. The dotted line shows the previous location of the boundary for ease of comparison. As above, the implied departure of the system from equilibrium excites Dungey-cycle convection in the magnetosphere and twin-vortex flow in the ionosphere, which is such as to transport closed flux tubes away from 
the merging gap region of closure sunward to other local times. The open-closed field line boundary thus contracts everywhere, as indicated by the large arrows. We note that in this situation, the foci of the twin-vortex flow cells will lie approximately at the ends of the nightside merging gap, as shown (and hence, at the ends of the substorm bulge, as observed), separating the equatorward flow of plasma across the nightside merging gap from the poleward flow in the adiaroic boundary region outside. In this case, therefore, where the nightside reconnection rate exceeds the dayside rate, the polar cap voltage determined from SuperDARN radar data will correspond essentially to the voltage across the nightside merging gap. Figure 10c shows the situation after the tail reconnection rate has declined once more and steady flows have been resumed. This occurs when the flux redistribution process is complete, such that (in the Cowley and Lockwood (1992) paradigm) the net closed flux is distributed approximately uniformly round the boundary, leaving the shape of the contracted boundary approximately unchanged.

In order to analyze this situation, specifically for the case of interest here, where the nightside reconnection rate exceeds the dayside rate, we write the amount of open flux present in the system as $\Phi(t)$, associated with a polar cap area $A(t)$, where $\Phi(t) \approx B_{i} A(t)$, and $B_{i}$ is the polar magnetic field strength (taken to be constant for simplicity and equal to $60000 \mathrm{nT}$ ). The dayside and nightside reconnection rates are then written as $\dot{\Phi}_{d}(t)$ and $\dot{\Phi}_{n}(t)$, where by definition $\dot{\Phi}_{d}(t)$ is positive (associated with increasing open flux), and $\dot{\Phi}_{n}(t)$ is negative (associated with decreasing open flux). The nightside reconnection rate can then be written as

$\dot{\Phi}_{n}(t)=-B_{i} \int_{s_{1}}^{s_{2}}\left(v_{n}(t)-v_{B}(t)\right) d s$,

where $v_{n}(t)$ is the plasma velocity normal to the boundary (taken as positive when directed equatorward), $v_{B}(t)$ is the boundary velocity (defined only in the normal direction and again taken as positive when directed equatorward), and $d s$ is the line element along the nightside merging gap of the open-closed field line boundary, from the dusk end of the line $\left(s_{1}\right)$ to the dawn end $\left(s_{2}\right)$. The integrand in Eq. (1) simply represents the transport of magnetic flux across the nightside merging gap in the latter's rest frame, per unit length of line. It is readily shown that the integral of the first term in Eq. (1) is

$$
B_{i} \int_{s_{1}}^{s_{2}} v_{n}(t) d s=V\left(s_{2}, t\right)-V\left(s_{1}, t\right) \approx V_{P C}(t),
$$

where $V\left(s_{1}, t\right)$ and $V\left(s_{2}, t\right)$ are the electric potentials at either end of the nightside merging gap, $V_{P C}(t)$ is the total transpolar voltage (as obtained from SuperDARN data), and the approximation holds when the nightside reconnection rate is larger than the dayside reconnection rate, as noted above. The integral of the second term in Eq. (1) is just the rate at which flux is swept out by the motion of the nightside reconnection line (i.e. the merging gap), taken as positive for equatorward motion, so that we have

$\dot{\Phi}_{n}(t) \approx-V_{P C}+B_{i} \frac{d A_{m g}}{d t}$.

Integrating these quantities over an event of duration $\tau$, and denoting averages by angle brackets, we then have

$\left\langle\dot{\Phi}_{n}\right\rangle \approx-\left\langle V_{P C}\right\rangle+B_{i} \frac{\Delta A_{m g}}{\tau}$,

where $\Delta A_{m g}$ is the total area swept out by the nightside merging gap during the event (positive for equatorward displacements). This equation thus determines the averaged nightside reconnection rate during an interval in terms of the mean polar cap voltage during the event and the displacement of the open-closed field line boundary in the region of the merging gap. The dayside reconnection rate is then given by the relation

$\left\langle\dot{\Phi}_{d}\right\rangle+\left\langle\dot{\Phi}_{n}\right\rangle=B_{i} \frac{\Delta A}{\tau}$,

where $\Delta A$ is the change in total area of the polar cap during the interval. Thus, introducing Eq. (4) into Eq. (5) yields

$\left\langle\dot{\Phi}_{d}\right\rangle=\left\langle V_{P C}\right\rangle+B_{i} \frac{\Delta A-\Delta A_{m g}}{\tau}$.

We now write $\Delta A_{m g} \approx f_{m g} \Delta A$, such that the area swept out by the nightside merging gap is some fraction $f_{m g}$ of the total change in area of the polar cap. From Eqs. (4) and (6) we then finally have

$\left\langle\dot{\Phi}_{n}\right\rangle \approx-\left\langle V_{P C}\right\rangle+f_{m g} B_{i} \frac{\Delta A}{\tau}$

and

$\left\langle\dot{\Phi}_{d}\right\rangle=\left\langle V_{P C}\right\rangle+\left(1-f_{m g}\right) B_{i} \frac{\Delta A}{\tau}$.

Clearly, if $\Delta A_{m g} \approx 0$ for a particular interval, then the magnitudes of the mean dayside and nightside reconnection rates are equal to each other, and also equal to the mean polar cap voltage $\left\langle V_{P C}\right\rangle$, as already noted above. On the other hand, if the polar cap area contracts during the interval such that $\Delta A<0$, then the magnitude of the nightside reconnection rate will be greater than $\left\langle V_{P C}\right\rangle$, while that of the dayside reconnection rate will be less than $\left\langle V_{P C}\right\rangle$. An inconsistency occurs if the magnitude of the second term in Eq. (7b) exceeds the first, implying that $\left\langle\dot{\Phi}_{d}\right\rangle$ is negative, contrary to the assumption. However, for the event analyzed here a more stringent condition on $\left\langle\dot{\Phi}_{d}\right\rangle$ can be considered, since we have argued above that the dayside reconnection rate probably remained in the range $30-45 \mathrm{kV}$ during the substorm. We will, therefore, impose this as a condition.

Here we will use Eqs. (7a) and (7b) to estimate the reconnection rates during the substorm expansion phase. If we examine these equations it is clear that some of the parameters on the right-hand sides are determined by the data better than others. In particular, the mean polar cap voltage $\left\langle V_{P C}\right\rangle$ 
during the intervals is reasonably well determined from the SuperDARN data, accepting the above interpretation of this quantity. On the other hand, the net area swept out by the open-closed field line boundary, $\Delta A$, and the fraction $f_{m g}$ of that associated with the nightside merging gap are not so well determined. With regard to $f_{m g}$, values can be estimated on the assumption that the effect of the flow is to redistribute the newly closed flux uniformly around the open-closed field line boundary, as in the Cowley and Lockwood (1992) paradigm. In this case $f_{m g}$ will also represent the fraction of the length of the open-closed field line boundary that is occupied by the nightside merging gap, and this can be estimated from the flow patterns during the expansion phase. We have shown above that the maximum and minimum in the electric potential will lie at either end of the nightside merging gap when the tail reconnection rate exceeds the dayside rate, as occurs here. Identification of the location of the foci of the flow cells thus provides an indication of the magnetic local time extent of the merging gap, from which $f_{m g}$ can be estimated. Examination of the flow maps during the expansion phase in Figs. 6 and 7 suggests $f_{m g} \sim 0.3$, and we employ a likely range $f_{m g}=0.2-0.4$. With regard to the change in polar cap area, we have been able to make only one approximate determination of the poleward displacement of the boundary, by $\sim 1^{\circ}$ latitude in the dusk sector where a clearly-defined flow reversal is evident. Our approach has been, therefore, to look carefully at the sensitivity of the results to these lesswell determined parameters.

In effect, what we have done is to plot contour maps of $\left\langle\dot{\Phi}_{n}\right\rangle$ and $\left\langle\dot{\Phi}_{d}\right\rangle$ determined from Eq. (7) on the $f_{m g}-\Delta A$ plane, and to delineate regions of consistency, both with the rather roughly-obtained limits on these latter parameters, and on $\left\langle\dot{\Phi}_{d}\right\rangle$ as discussed above. However, rather than use $\Delta A$ directly, we have used instead the latitude displacement of the boundary in the dusk local time sector. From this displacement the areas $\Delta A$ have been calculated by extrapolation of the initial and final boundary positions to other local times. Specifically, we have assumed for simplicity (and in accordance with the Cowley-Lockwood paradigm) that the initial and final boundaries are circles drawn about a common center. The position of the appropriate center has been judged from the shape of the flow patterns and auroral boundaries, and has been chosen on this basis to be displaced from the magnetic pole by $4^{\circ}$ of magnetic latitude along the 22 MLT meridian. The displacement of the center towards the nightside accommodates the usual day-night asymmetry in the position of the boundary, while the displacement towards dusk is suggested by the corresponding dawn-dusk asymmetry in the auroral boundary and is associated with the prevailing sense of IMF $B_{y}$ (negative). Examination of the flow and auroral patterns overall indicates that this is a very reasonable choice, and that no substantial improvement would result from attempting to use some more complex boundary shape.

Results are shown in Fig. 11, averaged over the interval of enhanced convection, i.e. over 00:44-01:08 UT (Fig. 8). For this interval we find $\left\langle V_{P C}\right\rangle \approx 59 \mathrm{kV}$ from the SuperDARN

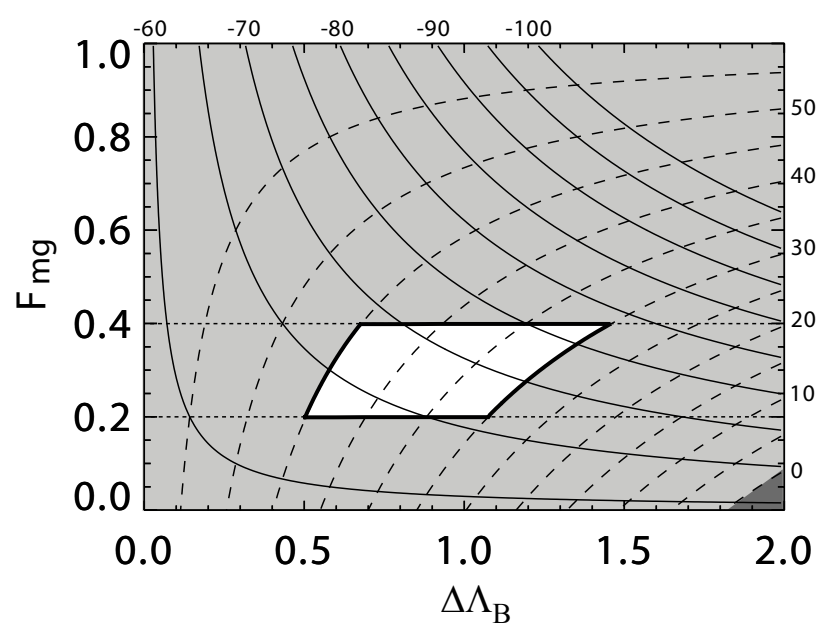

Fig. 11. Reconnection rate contours determined from Eq. (7) plotted on the $f_{m g}-\Delta \Lambda_{B}$ plane, where $f_{m g}$ is shown on the vertical axis, and the displacement of the magnetic latitude of the dusk flow reversal boundary $\Delta \Lambda_{B}$ during the substorm is shown on the horizontal axis. The solid lines show contours of the nightside reconnection rate obtained from Eq. (7a), while the dashed lines show contours of the dayside reconnection rate obtained from Eq. (7b). The dark-shaded area in the bottom right corner represents an area of inconsistent results in which the dayside reconnection rate becomes negative. The horizontal dotted lines mark the likely range of $f_{m g}$ values for the substorm, with the exterior region being shaded. Also shaded is the region of unlikely dayside reconnection rates lying outside the range $30-45 \mathrm{kV}$. The unshaded area corresponds to the region of solutions that conforms to both these constraints.

data. Using this value we have then plotted reconnection rate contours on the $f_{m g}-\Delta \Lambda_{B}$ plane, where $\left\langle\dot{\Phi}_{n}\right\rangle$ and $\left\langle\dot{\Phi}_{d}\right\rangle$ are shown by the solid and dashed lines, respectively. Parameter $f_{m g}$ is shown on the vertical axis, and the displacement in magnetic latitude of the dusk flow reversal boundary $\Delta \Lambda_{B}$ is shown on the horizontal axis. From the latter parameter we have calculated the change in area of the polar cap according to the above algorithm, starting from an initial boundary position at $\Lambda_{B}=77^{\circ}$ (Fig. 8), so that $\Delta A$ becomes increasingly negative as we move from left to right across the plot. We find that a $1^{\circ}$ poleward displacement of the boundary corresponds to a flux closure of $B_{i} \Delta A \approx-0.5 \times 10^{8} \mathrm{~Wb}$, while $2^{\circ}$ corresponds to $-0.9 \times 10^{8}$. At the left-hand edge of the plot we have $\Delta \Lambda_{B}=0$ (and $\Delta A=0$ ), so that at this limit Eqs. (7a) and (7b) give $\left\langle\dot{\Phi}_{d}\right\rangle=-\left\langle\dot{\Phi}_{n}\right\rangle=\left\langle V_{P C}\right\rangle$, as discussed above. We also have $\left\langle\dot{\Phi}_{n}\right\rangle=-\left\langle V_{P C}\right\rangle$ along the bottom edge of the plot, where $f_{m g}=0$, and $\left\langle\dot{\Phi}_{d}\right\rangle=\left\langle V_{P C}\right\rangle$ along the top edge of the plot, where $f_{m g}=1$. Away from these respective boundaries $\left\langle\dot{\Phi}_{n}\right\rangle$ becomes increasingly negative as $f_{m g}$ and $\Delta \Lambda_{B}$ both increase, while $\left\langle\dot{\Phi}_{d}\right\rangle$ drops to smaller values as $f_{m g}$ decreases and $\Delta \Lambda_{B}$ increases. Eventually $\left\langle\dot{\Phi}_{d}\right\rangle$ becomes negative in the dark-shaded area in the bottom right corner of the plot, this representing an area of inconsistent results (as discussed above) when $\left(1-f_{m g}\right) \Delta A$ becomes too negative. 
We now consider the likely ranges of the controlling parameters, and the consequent likely range of values of the reconnection rates. First, we indicated above that the likely range of $f_{m g}$ was between $\sim 0.2$ and 0.4. This range is marked by the horizontal dotted lines, and the exterior region is shown shaded. Second, according to our above arguments, we can reasonably expect the dayside reconnection rate to have lain in the range $\sim 30$ to $\sim 45 \mathrm{kV}$ during the interval. The regions outside these limits are also shaded. It can be seen that these two conditions delimit an area on the plot which spans a range of $\Delta \Lambda_{B}$ between $\sim 0.5^{\circ}$ and $\sim 1.5^{\circ}$. This result is, therefore, completely consistent with the $\mathrm{Su}-$ perDARN dusk boundary data, which indicate a poleward contraction by $\sim 1^{\circ}$, near the limit of resolution of the data. These latter observations do not, therefore, impose any further constraints in this case. The implication of the results in Fig. 11 is thus that the mean nightside reconnection rate during the substorm, averaged over the 24-min interval 00:4401:08 UT, was $\sim 70 \mathrm{kV}$, lying in the range $\sim 65-75 \mathrm{kV}$. Therefore, we further estimate that the total magnetic flux closed in the tail during the substorm was $\sim 0.9-1.1 \times 10^{8} \mathrm{~Wb}$, compared with $\sim 0.4-0.6 \times 10^{8} \mathrm{~Wb}$, which was opened on the dayside over the same interval. The net flux closed during the substorm was thus also $\sim 0.4-0.6 \times 10^{8} \mathrm{~Wb}$, corresponding to a $\sim 1^{\circ}$ contraction of the boundary overall. We also estimate the total amount of open flux present prior to substorm onset to have been $\sim 3 \times 10^{8} \mathrm{~Wb}$, this representing an entirely typical value under general conditions, and for presubstorm intervals in particular (Newell et al., 2001). Thus, the net flux closed during the substorm represents $\sim 15-20 \%$ of the total open flux present prior to onset.

\section{Summary}

The main points which we wish to emphasise are as follows.

(a) Ionospheric flow was excited in concert with the onset and decay of the expansion phase of an isolated high-latitude substorm of modest amplitude and duration. The flow was of twin-vortex form with foci on the nightside at either end of the substorm auroral bulge. The transpolar voltage associated with the flow increased from $\sim 40 \mathrm{kV}$ prior to onset, to peak at $\sim 80 \mathrm{kV}$ after $15 \mathrm{~min}$, which was $\sim 5 \mathrm{~min}$ after the peak in the magnetic disturbance. The flow then declined back down to $\sim 35 \mathrm{kV}$ over $\sim 10 \mathrm{~min}$ in the recovery phase. During this interval the IMF remained steady, with $B_{y} \approx-9 \mathrm{nT}$, and $B_{z} \approx+5 \mathrm{nT}$. The flow enhancement (and substorm) is inferred to have resulted from a burst of reconnection in the pre-midnight plasma sheet.

(b) The return flows of the enhanced flow pattern extended widely on either side of the region directly effected by the substorm, over the polar cap, and into the dayside sector. The changed and enhanced flow pattern, combined with prevailing ionospheric conductivities, produced magnetic disturbances of modest amplitude over a large area surrounding the substorm-disturbed region. These data thus support the prior conclusions of Grafe et al. (1987) and Opgenoorth and
Pellinen (1998), based principally on magnetic data from the dusk sector, that a prompt response in the global convection system often occurs at expansion phase onset. Opgenoorth and Pellinen suggested that the response could be produced by a suppression of the flow in the region of dipolarized flux in the nightside magnetosphere, mapping essentially to the substorm bulge, resulting in a deflection of the surrounding flow around this region. Such deflection would give rise to a flow enhancement on either side of the bulge, but this effect alone would result in no change in the overall transpolar voltage. For the localized substorm studied here, however, we have found that the principal effect was the excitation of new voltage during the expansion phase, in which the total transpolar voltage increased by a factor of two.

(c) Flow suppression within and deflection around the dipolarized region (the blob of brightest auroral emission) occurred during the expansion phase, as observed in previous studies by Morelli et al. (1995), Yeoman et al. (2000), and Khan et al. (2001). However, in the present case, the region effected was relatively small and localized near midnight, such that this represented a distinct but secondary effect. The main flow effect in this particular substorm was thus the overall enhancement mentioned above.

(d) The nightside reconnection rate averaged over the 24-min substorm interval of enhanced flows is estimated to have been $\sim 65-75 \mathrm{kV}$, compared with continuing dayside reconnection rates of $\sim 30-45 \mathrm{kV}$. The net destruction of open flux during the substorm is thus estimated to have been $\sim 0.4-0.6 \times 10^{8} \mathrm{~Wb}$, which corresponds to $\sim 15-20 \%$ of the open flux present at onset. The corresponding overall contraction of the open-closed field line boundary during the substorm was thus $\sim 1^{\circ}$ latitude, close to the resolution limit of the SuperDARN data.

The explanation we have forwarded for the flow effect observed here, in terms of the contribution of tail reconnection and field dipolarization to Dungey cycle convection, should hold for substorms in general. We may, therefore, expect that such flow excitation should be a common feature of substorm intervals. Factors which might mitigate against a clear identification of the effect in general cases include (a) variations in the direction and strength of the IMF during the substorm, which produce additional modulations of the flow driven by magnetopause reconnection; (b) large-scale flow reconfigurations on the nightside due to flow suppression and deflection effects over larger areas of the substorm bulge; (c) dropout of radar backscatter from the crucial substormdisturbed region due to ionospheric absorption; and (d) inadequate imaging of the flow pattern within the collective field of view of the radar array, which is limited both in local time and in latitude. The identification of the flow enhancement effect in the present data set clearly resulted from a rather fortunate combination of geophysical and operational circumstances. A future agenda is set, however, to search for related features in other substorms.

Acknowledgements. We would like to thank the PIs of the SuperDARN radars for provision of the radar data employed in this 
study. The data employed were from radars funded by the research funding agencies of Canada, France, the UK, and USA. We would also like to thank Dr Mike Ruohoniemi of the Johns Hopkins University Applied Physics Laboratory for provision of the Map-Potential algorithm software. For the ground magnetometer data, we thank the Finnish Meteorological Institute and its co-institutes who maintain the IMAGE magnetometer array, the Canadian Space Agency who constructed and maintain the CANOPUS instrument array, the Geological Survey of Canada (Natural Resources Canada) for use of data from its magnetometer array, Boston University, Augsburg College, the University of Alberta and the Geological Survey of Canada who run the MACCS magnetometer array (which is supported by the National Science Foundation's Magnetospheric Physics Program), and Ian Mann and David Milling for the SAMNET data. SAMNET is a PPARC National Facility deployed and operated by the University of York. We would also like to thank Howard Singer and the National Oceanic and Atmospheric Administration for GOES-8 magnetic field data, Geoff Reeves and the Los Alamos National Laboratory for LANL particle data, Norman Ness and Charles Smith for provision of ACE magnetometer data, and David McComas for provision of ACE SWEPAM data. AG was supported during this study by a PPARC Quota Studentship, and SWHC by PPARC Senior Fellowship PPA/N/S/2000/00197. SuperDARN studies and operations at the University of Leicester are supported by PPARC grants PPA/G/O/1999/00181 and PPA/R/R/1997/00256 respectively.

The Editor in Chief thanks R. L. McPherron and another referee for their help in evaluating this paper.

\section{References}

Angelopoulos, V., Baumjohann, W., Kennel, C. F., Coroniti, F. V., Kivelson, M. G., Pellat, R., Walker, R. J., Lühr, H., and Paschmann, G.: Bursty bulk flows in the central plasma sheet, J. Geophys. Res., 97, 4027, 1992.

Baker, K. B. and Wing, S.: A new magnetic coordinate system for conjugate studies at high latitudes, J. Geophys. Res., 94, 9139, 1989.

Baker, D. N., Pulkkinen, T. I., Angelopoulos, V., Baumjohann, W., and McPherron, R. L.: Neutral line model of substorms: Past results and present view, J. Geophys. Res., 101, 12 975, 1996.

Baumjohann, W., Paschmann, G., and Lühr, H.: Characteristics of high-speed flows in the plasma sheet, J. Geophys. Res., 95, 3801, 1990.

Belian, R. D., Gisler, G. R., Cayton, T., and Christensen, R.: High$\mathrm{Z}$ energetic particles at geostationary orbit during the great solar proton event series of October 1989, J. Geophys. Res., 97, $16897,1992$.

Blanchard, G. T., Lyons, L. R., de la Beaujardire, O., Doe, R. A., and Mendillo, M.: Measurements of the magnetotail reconnection rate, J. Geophys. Res., 101, 15 265, 1996.

Blanchard, G. T., Lyons, L. R., and de la Beaujardire, O.: Magnetotail reconnection rate during magnetospheric substorms, J. Geophys. Res., 102, 24 303, 1997.

Cowley, S. W. H.: Excitation of flow in the Earth's magnetosphereionosphere system: observations by incoherent-scatter radar, in 'Polar Cap Boundary Phenomena' edited by A. Egeland, J. Moen, and M. Lockwood, Kluwer Academic Publ., Dordrecht, 127, 1998.

Cowley, S. W. H. and Lockwood, M.: Excitation and decay of solar wind-driven flows in the magnetosphere-ionosphere system,
Ann. Geophysicae, 10, 103, 1992.

Cowley, S. W. H. and Lockwood, M.: Time-dependent flows in the coupled solar wind- magnetosphere-ionosphere system, Adv. Space Res., 18, (8)141, 1996.

Cowley, S. W. H., Khan, H., and Stockton-Chalk, A.: Plasma flow in the coupled magnetosphere- ionosphere system and its relationship to the substorm cycle, in 'Substorms-4' edited by S. Kokobun and Y. Kamide, Terra Sci. Publ. Co., Tokyo, 623, 1998.

Cowley, S. W. H., Morelli, J. P., Freeman, M. P., Lockwood, M., and Smith, M. F.: Excitation and decay of flows in the magnetosphere-ionosphere system due to magnetic reconnection at the dayside magnetopause and in the geomagnetic tail, in 'Substorms 1', ESA SP-335, ESA, Noordwijk, The Netherlands, 117, 1992.

de la Beaujardire, O., Lyons, L. R., and Friis-Christensen, E.: Sondrestrom radar measurements of the reconnection electric field, J. Geophys. Res., 96, 13 907, 1991.

de la Beaujardire, O., Lyons, L. R., Ruohoniemi, J. M., FriisChristensen, E., Danielsen, C., Rich, F. J., and Newell, P. T.: Quiet-time intensifications along the poleward boundary near midnight, J. Geophys. Res., 99, 287, 1994.

Elphic, R. C., Lockwood, M., Cowley, S. W. H., and Sandholt, P. E.: Flux transfer events at the magnetopause and in the ionosphere, Geophys. Res. Lett., 17, 2241, 1990.

Etemadi, A., Cowley, S. W. H., Lockwood, M., Bromage, B. J. I., Willis, D. M., and Lühr, H.: The dependence of high-latitude dayside ionospheric flows on the north-south component of the IMF: a high-time resolution correlation analysis using EISCAT "Polar" and AMPTE UKS and IRM data, Planet. Space Sci., 36, 471, 1988.

Fox, N. J., Cowley, S. W. H., Davda, V. N., Enno, G., FriisChristensen, E., Greenwald, R. A., Hairston, M. R., Kivelson, M. G., Lester, M., Lockwood, M., Lühr, H., Milling, D. K., Murphree, J. S., Pinnock, M., and Reeves, G. D.: A multipoint study of a substorm occurring on 7 December 1992 and its theoretical implications, Ann. Geophysicae, 17, 1369, 1999.

Frank, L. A. and Sigwarth, J. B.: Findings concerning the positions of substorm onsets with auroral images from the Polar spacecraft, J. Geophys. Res., 105, 12 747, 2000.

Frank, L. A., Sigwarth, J. B., Craven, J. D., Cravens, J. P., Dolan, J. S., Dvorsky, M. R., Hardebeck, P. K., Harvey, J. D., and Muller, D. W.: The Visible Imaging System (VIS) for the Polar spacecraft, Space Sci. Rev., 71, 297, 1995.

Freeman, M. P. and Southwood, D. J.: The effect of magnetospheric erosion on mid- and high-latitude ionospheric flows, Planet. Space Sci., 36, 509, 1988.

Friedrich, E., Samson, J. C., Voronkov, I., and Rostoker, G.: Dynamics of the substorm expansion phase, J. Geophys. Res., 106, $13145,2001$.

Friis-Christensen, E., Kamide, Y., Richmond, A. D., and Matsushita, S.: Interplanetary magnetic field control of high-latitude electric fields and currents determined from Greenland magnetometer data, J. Geophys. Res., 90, 1325, 1985.

Grafe, A., Pellinen, R. J., Baumjohann, W., and Vallinkoski, M.: Development of the auroral electrojets on March 16, 1978: An event study, Geophysica, 23, 113, 1987.

Grant, I. F., McDiarmid, D. R., and McNamara, A. G.: A class of high-m pulsations and its auroral radar signature, J. Geophys. Res., 97, 8439, 1992.

Greenwald, R. A., Baker, K. B., Dudeney, J. R., Pinnock, M., Jones, T. B., Thomas, E. C., Villain, J.-P., Cerisier, J.-C., Senior, C., Hanuise, C., Hunsucker, R. D., Sofko, G., Koehler, J., 
Nielsen, E., Pellinen, R., Walker, A. D. M., Sato, N., and Yamagishi, H.: DARN/SuperDARN: A global view of the dynamics of high-latitude convection, Space Sci. Rev., 71, 761, 1995.

Grocott, A., Cowley, S. W. H., and Davies, J. A.: Comparison of ionospheric electric currents and plasma convection patterns observed during substorms, in 'Proc. Fifth Internat. Conf. on Substorms', ESA SP-443, ESA, Noordwijk, The Netherlands, 295, 2000.

Henderson, M. G., Reeves, G. D., and Murphree, J. S.: Are northsouth aligned auroral structures an ionospheric manifestation of bursty bulk flows?, Geophys. Res. Lett., 25, 3737, 1998.

Hones, E. W., Jr.: Transient phenomena in the magnetotail and their relation to substorms, Space Sci. Rev., 23, 393, 1979.

Hsu, T. S. and McPherron, R. L.: Occurrence frequency of substorm field and plasma signatures observed near-Earth by ISEE-1/2, in 'Proc. Third Internat. Conf. on Substorms', ESA SP-389, ESA, Noordwijk, The Netherlands, 333, 1996.

Hughes, W. J. and Engebretson, M. J.: MACCS: Magnetometer Array for Cusp and Cleft Studies, in Satellite-Ground Based Coordination Sourcebook, edited by M. Lockwood, M. N. Wild, H. J. Opgenoorth, ESA-SP-1198, 119, 1997.

Khan, H. and Cowley, S. W. H.: Observations of the response time of high latitude ionospheric convection to variations in the interplanetary magnetic field using EISCAT and IMP-8 data, Ann. Geophysicae, 17, 1306, 1999.

Khan, H., Cowley, S. W. H., Kolesnikova, E., Lester, M., Brittnacher, M. J., Hughes, T. J., Hughes, W. J., Kurth, W. S., McComas, D. J., Newitt, L., Owen, C. J., Reeves, G. D., Singer, H. J., Smith, C. W., Southwood, D. J., and Watermann, J. F.: Observations of two complete substorm cycles during the Cassini Earth swing-by: Cassini magnetometer data in a global context, J. Geophys. Res., in press, 2001.

Kirkwood, S., Opgenoorth, H. J., and Murphree, J. S.: Ionospheric conductivities, electric fields and currents associated with auroral substorms measured by the EISCAT radar, Planet. Space Sci., 36, 1359, 1988.

Lockwood, M., Moen, J., Cowley, S. W. H., Farmer, A. D., Løvhaug, U. P., Lühr, H., and Davda, V. N.: Variability of dayside convection and motions of the cusp/cleft aurora, Geophys. Res. Lett., 20, 1011, 1993.

Lockwood, M., Sandholt, P. E., Cowley, S. W. H., and Oguti, T.: Interplanetary field control of dayside auroral activity and the transfer of momentum across the dayside magnetopause, Planet. Space Sci., 37, 1347, 1989.

Lu, G., Cowley, S. W. H., Milan, S. E., Sibeck, D. G., Greenwald, R. A., and Moretto, T.: Solar wind effects on ionospheric convection: A review, J. Atmos. Solar-Terr. Phys., in press, 2001.

Lühr, H., Aylward, A., Bucher, S. C., Pajunpaa, A., Pajunpaa, K., Holmboe, T., and Zalewski, S. M.: Westward moving dynamic substorm features observed with the IMAGE magnetometer network and other ground-based instruments, Ann. Geophysicae, 16, 425, 1998.

Lui, A. T. Y.: A synthesis of magnetospheric substorm models, J. Geophys. Res., 96, 1849, 1991.

Lui, A. T. Y.: Current disruption in the Earth's magnetosphere: Observations and models, J. Geophys. Res., 101, 13 067, 1996.

Lyons, L. R., Nagai, T., Blanchard, G. T., Samson, J. C., Yamamoto, T., Mukai, T., Nishida, A., and Kokubun, S.: Association between Geotail plasma flows and auroral poleward boundary intensifications observed by CANOPUS photometers, J. Geophys. Res., 104, 4485, 1999.

Machida, S., Miyashita, Y., Ieda, A., Nishida, A., Mukai, T.,
Saito, Y., and Kokubun, S.: Geotail observations of flow velocity and north-south magnetic field variations in the near and middistant tail associated with substorm onsets, Geophys. Res. Lett., 26, 635, 1999.

McComas, D. J., Bame, S. J., Barker, P., Feldman, W. C., Phillips, J. L., Riley, P., and Griffee, J. W.: Solar Wind Electron Proton Alpha Monitor (SWEPAM) for the Advanced Composition Explorer, Space Sci. Rev., 86, 563, 1998.

McPherron, R. L.: Growth phase of magnetospheric substorms, J. Geophys. Res., 75, 5592, 1970.

Milan, S. E., Jones, T. B., Lester, M., Warrington, E. M., and Reeves, G. D.: Substorm correlated absorption on a $3200 \mathrm{~km}$ trans-auroral HF propagation path, Ann. Geophysicae, 14, 182, 1996.

Milan, S. E., Lester, M., Cowley, S. W. H., and Brittnacher, M.: Convection and auroral response to a southward turning of the IMF: Polar UVI, CUTLASS, and IMAGE signatures of transient magnetic flux transfer at the magnetopause, J. Geophys. Res., $105,15741,2000$.

Moen, J., Sandholt, P. E., Lockwood, M., Denig, W. F., Løvhaug, U. P., Lybekk, B., Egeland, A., Opsvik, D., and FriisChristensen, E.: Events of enhanced convection and related dayside auroral activity, J. Geophys. Res., 100, 23 917, 1995.

Morelli, J. P., Bunting, R. L., Cowley, S. W. H., Farrugia, C. J., Freeman, M. P., Friis-Christensen, E., Jones, G. O. L., Lester, M., Lewis, R. V., Lühr, H., Orr, D., Pinnock, M., Reeves, G. D., Williams, P. J. S., and Yeoman, T. K.: Radar observations of auroral zone flows during a multiple-onset substorm, Ann. Geophysicae, 13, 1144, 1995.

Nagai, T. and Machida, S.: Magnetic reconnection in the near-earth magnetotail, in 'New Perspectives on the Earth's Magnetotail' edited by A. Nishida, D. N. Baker and S. W. H. Cowley, Geophys. Mono. 105, AGU, Washington, DC, 211, 1998.

Nagai, T., Fujimoto, M., Saito, Y., Machida, S., Teresawa, T., Nakamura, R., Yamamoto, T., Mukai, T., Nishida, A., and Kokubun, S.: Structure and dynamics of magnetic reconnection for substorm onsets with Geotail observations, J. Geophys. Res., 103, $13067,1998$.

Neudegg, D. A., Cowley, S. W. H., Milan, S. E., Yeoman, T. K., Lester, M., Provan, G., Haerendel, G., Baumjohann, W., Nikutowski, B., Büchner, J., Auster, U., Fornacon, K.-H., and Georgescu, E.: A survey of magnetopause FTEs and associated flow bursts in the polar ionosphere, Ann. Geophysicae, 18, 416, 2000.

Neudegg, D. A., Yeoman, T. K., Cowley, S. W. H., Provan, G., Haerendel, G., Baumjohann, W., Auster, U., Fornacon, K.-H., Georgescu, E., and Owen, C. J.: A flux transfer event observed at the magnetopause by the Equator-S spacecraft and in the ionosphere by the CUTLASS HF radar, Ann. Geophysicae, 17, 707, 1999.

Newell, P. T., Liou, K., Sotirelis, T., and Meng, C.-I.: Polar Ultraviolet Imager observations of global auroral power as a function of polar cap size and magnetotail stretching, J. Geophys. Res., 106, 5895, 2001.

Opgenoorth, H. J. and Pellinen, R. J.: The reaction of the global convection electrojets to the onset and expansion of the substorm current wedge, in: 'Substorms-4' (Eds) by Kokobun, S. and Kamide, Y., Terra Sci. Publ. Co., Tokyo, 663, 1998.

Petrukovich, A. A., Sergeev, V. A., Zleneyi, L. M., Mukai, T., Yamamoto, T., Kokubun, S., Shiokawa, K., Deehr, C. S., Budnick, E. Y., Büchner, J., Fedorov, A. O., Grigorieva, V. P., Hughes, T. J., Pissarenko, N. F., Romanov, S. A., and Sandahl, I.: 
Two spacecraft observations of a reconnection pulse during an auroral breakup, J. Geophys. Res., 103, 47, 1998.

Pinnock, M., Rodger, A. S., Dudeney, J. R., Baker, K. B., Greenwald, R. A., and Greenspan, M.: Observations of an enhanced convection channel in the cusp ionosphere, J. Geophys. Res., 98, 3767, 1993.

Pinnock, M., Rodger, A. S., Dudeney, J. R., Rich, F., and Baker, K. B.: High temporal and spatial observations of the ionospheric cusp, Ann. Geophysicae, 13, 919, 1995.

Provan, G., Yeoman, T. K., and Milan, S. E.: CUTLASS Finland radar observations of the ionospheric signatures of flux transfer events and the resulting plasma flows, Ann. Geophysicae, 16, 1411, 1998.

Reeves, G. D., Belian, R. D., and Fritz, T. A.: Numerical tracing of energetic particle drifts in a model magnetosphere, J. Geophys. Res., 96, 13 997, 1991.

Ridley, A. J., Lu, G., Clauer, C. R., and Papitashvili, V. O.: A statistical study of the ionsopheric convection response to changing interplanetary magnetic field conditions using the assimilative mapping of ionospheric electrodynamics technique, J. Geophys. Res., 103, 4023, 1998.

Ruohoniemi, J. M. and Baker, K. B.: Large-scale imaging of highlatitude convection with Super Dual Auroral Radar Network HF radar observations, J. Geophys. Res., 103, $20797,1998$.

Ruohoniemi, J. M. and Greenwald, R. A.: Statistical patterns of high-latitude convection obtained from Goose Bay HF radar observations, J. Geophys. Res., 101, 21 743, 1996.

Ruohoniemi, J. M., Greenwald, R. A., de la Beaujardire, O., and Lester, M.: The response of the high-latitude dayside ionosphere to a sudden southward IMF turning, Ann. Geophysicae, 11, 544, 1993.

Sergeev, V. A., Aulamo, O. A., Pellinen, R. J., Vallinkoski, M. K., Bsinger, T., Cattell, C. A., Elphic, R. C., and Williams, D. J.: Non-substorm transient injection events in the ionosphere and magnetosphere, Planet. Space Sci., 38, 231, 1990.

Sergeev, V. A., Liou, K., Meng, C.-I., Newell, P. T., Brittnacher, M., Parks, G., and Reeves, G. D.: Development of auroral streamers in association with localized impulsive injections to the inner magnetotail, Geophys. Res. Lett., 26, 417, 1999.

Sergeev, V. A., Sauvaud, J.-A., Popescu, D., Liou, K., Newell, P. T., Brittnacher, M., Parks, G., Nakamura, R., Mukai, T., and Reeves, G. D.: Multiple spacecraft observation of a narrow transient plasma jet in the Earth's plasma sheet, Geophys. Res. Lett., $27,851,2000$.

Shepherd, S. G. and Ruohoniemi, J. M.: Electrostatic potential patterns in the high-latitude ionosphere constrained by SuperDARN measurements, J. Geophys. Res., 105, 23 005, 2000.

Singer, H. J., Matheson, L., Grubb, R., Newman, A., and Bouwer, S. D.: Monitoring space weather with GOES Magnetometers, SPIE Proceedings, 2812, 1996.

Siscoe, G. L. and Huang, T. S.: Polar cap inflation and deflation, J. Geophys. Res., 90, 543, 1985.

Smith, C. W., Acuña, M. H., Burlaga, L. F., L'Heureux, J., Ness, N. F., and Scheifele, J.: The ACE Magnetic Field Experiment, Space Sci. Rev., 86, 613, 1999.

Stone, E. C., Frandsen, A. M., Mewaldt, R. A., Christian, E. R., Margolies, D., Ormes, J. F., and Snow, F.: The Advanced Composition Explorer, Space Sci. Rev., 86, 1, 1998.

Todd, H., Cowley, S. W. H., Lockwood, M., Willis, D. M., and Lühr, H.: Response time of the high-latitude dayside ionosphere to sudden changes in the north-south component of the IMF, Planet. Space Sci., 36, 1415, 1988.

Tsyganenko, N. A.: A magnetospheric magnetic field model with a warped tail current sheet, Planet. Space Sci., 37, 5, 1989.

Watanabe, M., Pinnock, M., Rodger, A. S., Sato, N., Yamagishi, H., Yukimatu, A. S., Greenwald, R. A., Villain, J.-P., and Hairston, M. R.: Localised activation of the distant tail neutral line just prior to substorm onsets, J. Geophys. Res., 103, 17651 , 1998.

Weimer, D. R.: Substorm influence on the ionospheric electric potentials and currents, J. Geophys. Res., 104, 185, 1999.

Wild, J. A., Cowley, S. W. H., Davies, J. A., Khan, H., Lester, M., Milan, S. E., Provan, G., Yeoman, T. K., Balogh, A., Dunlop, M. W., Fornacon, K.-H., and Georgescu, E.: First simultaneous observations of flux transfer events at the high-latitude magnetopause by the Cluster spacecraft and pulsed radar signatures in the conjugate ionosphere by the CUTLASS and EISCAT radars, Ann. Geophysicae, 10-12, 1491, 2001.

Yeoman, T. K. and Lühr, H.: CUTLASS/IMAGE observations of high-latitude convection features during substorms, Ann. Geophysicae, 15, 692, 1997.

Yeoman, T. K., Milling, D. K., and Orr, D.: Pi2 pulsation polarization patterns on the U.K. Sub- auroral Magnetometer Network (SAMNET), Planet Space Sci., 38, 589, 1990.

Yeoman, T. K., Mukai, T., and Yamamoto, T.: Simultaneous ionospheric and magnetospheric observations of azimuthally propagating transient features during substorms, Ann. Geophysicae, 16, 754, 1998

Yeoman, T. K., Davies, J. A., Wade, N. M., Provan, G., and Milan, S. E.: Combined CUTLASS, EISCAT and ESR observations of ionospheric plasma flows at the onset of an isolated substorm, Ann. Geophysicae, 18, 1073, 2000 